\title{
Metal-dependant stereoselectivity in the Pauson-Khand cyclization of $N$-propargyl- $\gamma$-amino vinyl sulfones
}

\author{
Anna Picó and Albert Moyano* \\ Unitat de Recerca en Sintesi Asimètrica, Departament de Química Orgànica, Universitat de \\ Barcelona. Facultat de Química, C. Martí i Franquès 1-11,08028-Barcelona, Catalonia, Spain \\ E-mail: amoyano@ub.edu
}

Dedicated to Prof. Joan Bosch on the occasion of his 60th birthday

\begin{abstract}
An efficient enantioselective route to $N$-propargyl- $\gamma$-amino vinyl sulfones has been developed, and their Pauson-Khand cyclization has been investigated. The stereoselectivity of the reaction depends both on the structure of the substrate and on the nature of the metal-carbonyl promoter.
\end{abstract}

Keywords: $\gamma$-Amino vinyl sulfones, azabicyclo[3.3.0]octenones, Pauson-Khand reaction

\section{Introduction}

The intramolecular version of the Pauson-Khand (PK) reaction, ${ }^{1}$ a metal-mediated $[2+2+1]$ formal cycloaddition of an alkyne, an alkene and carbon monoxide to give a 2-cyclopentenone unit, has been widely utilized in the direct construction of carbo- and heterobicyclic systems, often with high degrees of stereocontrol. ${ }^{2}$ While the presence of electron-deficient substituents in the alkene moiety leads in most instances to low yields and/or to the formation of conjugated 1,3-dienes, ${ }^{3}$ Carretero and co-workers have demonstrated that 1-phenylsulfonyl-3-oxygenated enynes are excellent substrates for the reaction. ${ }^{4}$ Moreover, contrary to the standard selectivity of allyl substituted enynes in PK cyclizations, in which the allylic substituent is located preferentially in the less hindered exo face of the bicyclic product, ${ }^{1,5}$ the intramolecular PK reaction of several $\gamma$-alkoxy vinyl sulfones takes place with high endo selectivities.

In the context of our long-standing interest in the synthetic applications of scalemic 2-(1aminoalkyl)oxiranes, ${ }^{6}$ we have recently developed a general, high-yielding and enantioselective route to $\gamma$-amino vinyl sulfones. ${ }^{7}$ In spite of their high biomedical relevance as key components of potent and selective cysteine protease inhibitors, ${ }^{8}$ practically nothing is known about the chemistry of these compounds. We decided therefore to investigate the PK cyclization of $N$ propargyl- $\gamma$-amino vinyl sulfones, paying special attention to the stereochemical outcome of the 
process. Herein, we report the results of these studies, which have disclosed an unusual dependence of the exo/endo selectivity of the reaction on the nature of the metal promoter.

\section{Results and Discussion}

In order to develop a general and efficient process for the synthesis of $N$-propargyl- $\gamma$-amino vinyl sulfones, our initial goal was the preparation of the specific enyne 2a starting from the $N$ Boc protected $\gamma$-amino vinyl sulfone 1a, which we had previously synthesized in our laboratory. ${ }^{7}$ This transformation, however, proved to be more challenging than anticipated. In effect, the attempted $N$-propargylation of $\mathbf{1 a}$ by treatment with sodium hydride and propargyl bromide in anhydrous tetrahydrofuran resulted in the irreversible elimination of the phenylsulfinate anion, and the diene 3a was the sole product isolated from the reaction mixture (Scheme 1). Likewise, all attempts to effect the $N$-propargylation of the epoxide 4a (a synthetic precursor of $\mathbf{1 a})^{7}$ were unsuccessful. Better results were obtained by trifluoroacetic acid-mediated cleavage of the Boc group in 1a, followed by reaction of the intermediate trifluoroacetate salt with propargyl bromide in the presence of potassium carbonate. In this way, the unprotected enyne 2'a was obtained in an overall $60 \%$ yield after chromatographic purification. We were disappointed to find that this yield dropped dramatically during the scaling up of this process, and we devoted considerable effort to develop a more practical alternative.
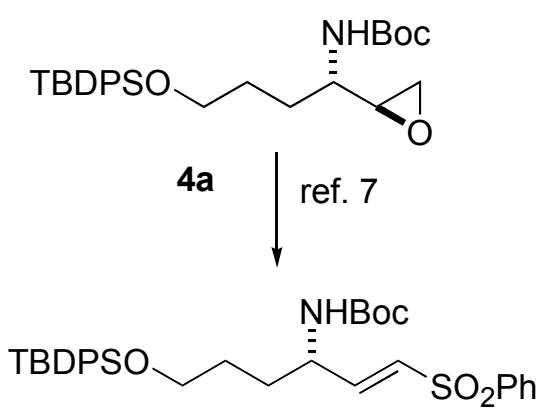

$1 a$

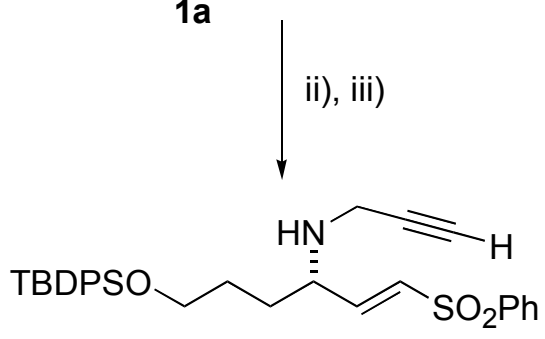

2'a
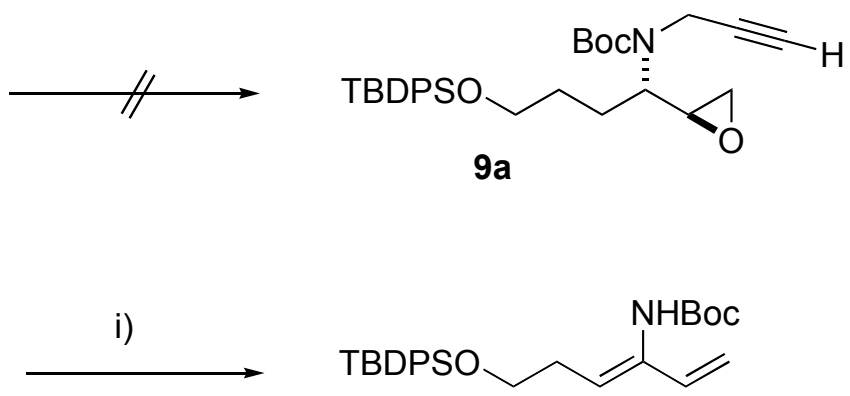

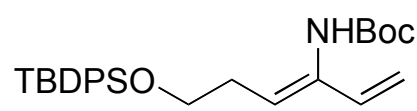

$3 a$

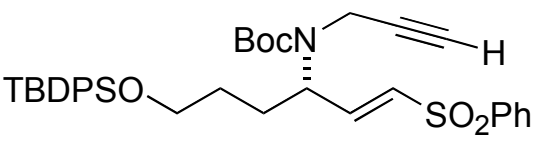

$2 a$

Scheme 1. i) $\mathrm{NaH}$, propargyl bromide, THF, $\Delta$ (66\% yield); ii) TFA, $\mathrm{CH}_{2} \mathrm{Cl}_{2}$, rt; iii) propargyl bromide, $\mathrm{K}_{2} \mathrm{CO}_{3}$, DMF, rt (60\% yield from 1a). 
After some experimentation, we decided to replace the Boc protecting group by a Cbz one, taking into account some observations previously made by Jeong et al. ${ }^{9}$ In this way, we prepared the $\mathrm{N}$-Cbz epoxide $\mathbf{4 b}$ and the corresponding $\gamma$-amino vinyl sulfone $\mathbf{1 b}$, starting from the known ${ }^{7}$ azido diol 5, by the synthetic route depicted in Scheme 2, that takes place with good overall yield.<smiles>COS(=O)(=O)OCCCC(N)C(O)CO</smiles>

5

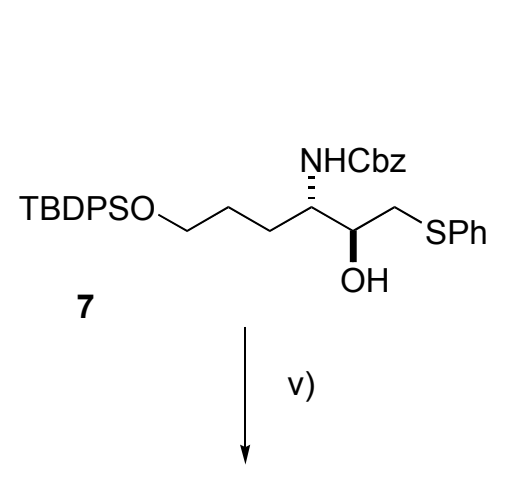<smiles>CCOC(=O)N[C@@H](CCCOS(=O)(=O)O)C(O)COS(=O)(=O)c1ccccc1</smiles>

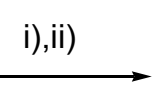

iv)<smiles>CCCCOCCCC(NC(=O)OCc1ccccc1)C(O)CO</smiles>

iii)

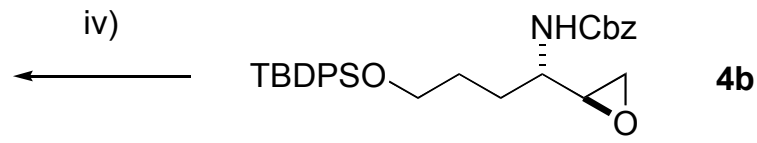

b

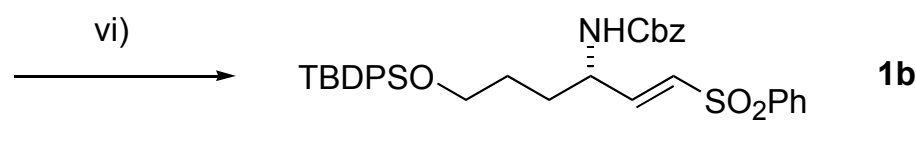

Scheme 2. i) $\mathrm{H}_{2}$, cat. $10 \% \mathrm{Pd} / \mathrm{C}, \mathrm{MeOH}$, rt; ii) $\mathrm{PhCH}_{2} \mathrm{OCOCl}, \mathrm{Et}_{3} \mathrm{~N}, 4-\mathrm{DMAP}, \mathrm{THF}, \mathrm{rt}(65 \%$ yield, two steps); iii) $\mathrm{Ph}_{3} \mathrm{P}$, DIAD, $\mathrm{CH}_{2} \mathrm{Cl}_{2}$, reflux (89\% yield); iv) $\mathrm{PhSH}, \mathrm{Et}_{3} \mathrm{~N}, \mathrm{MeOH}$, reflux (91\% yield); v) m-CPBA, $\mathrm{CH}_{2} \mathrm{Cl}_{2}$, rt (95\% yield); vi) $\mathrm{MsCl}$, 4-DMAP, $\mathrm{CH}_{2} \mathrm{Cl}_{2}$, rt (75\% yield).

As it happened in the case of $\mathbf{1 a}$, the base-mediated $N$-propargylation of $\mathbf{1 b}$ was unsuccessful, leading to the formation of the diene $\mathbf{3 b}$. We were however pleased to find that, contrary to the case of $\mathbf{4 a}$ (see Scheme 1), epoxide $\mathbf{4 b}$ could be $N$-propargylated in good yield (Scheme 3). 


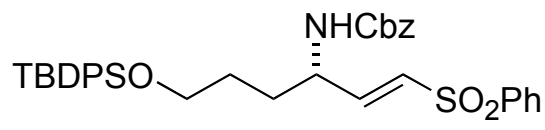

$1 \mathrm{~b}$<smiles>O=C([OH2+])N[C@@H](CCCOS(=O)(=O)c1ccccc1)C1CO1</smiles>

$4 b$

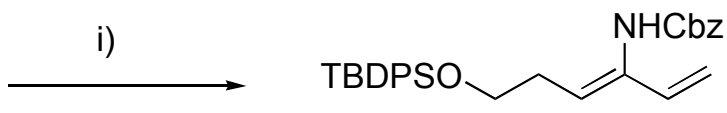

$3 b$

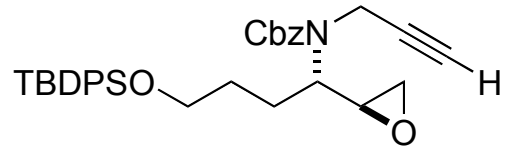

$9 b$

Scheme 3. i) $\mathrm{NaH}$, propargyl bromide, THF or DMF, $\Delta$ (50\% yield); ii) $\mathrm{NaH}$, propargyl bromide, THF-HMPA, $\Delta$ ( $82 \%$ yield $)$.

Starting from $\mathbf{9 b}$, the target enyne $\mathbf{2 b}$ could now be accessed in excellent overall yield, as shown in Scheme 4.

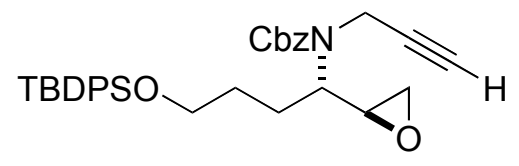

$9 b$

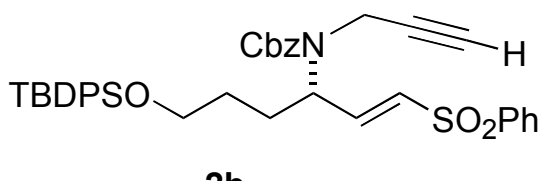

2b

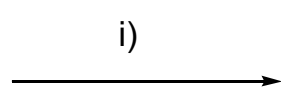

iii)

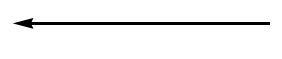

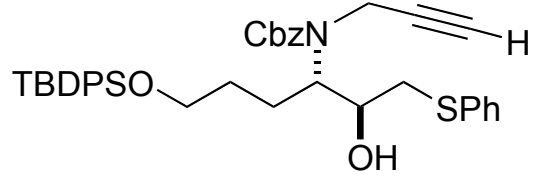

$10 \mathrm{~b}$

ii)

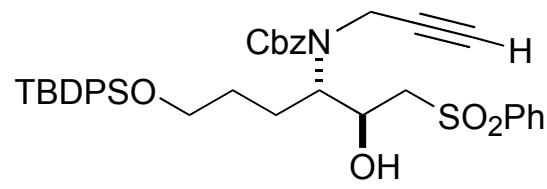

$11 b$

Scheme 4. i) $\mathrm{PhSH}, \mathrm{Et}_{3} \mathrm{~N}, \mathrm{MeOH}$, reflux (93\% yield); ii) $m$-CPBA, $\mathrm{CH}_{2} \mathrm{Cl}_{2}, \mathrm{rt}$ (95\% yield); iii) $N$-cyclohexyl-( $N$ '-morpholinoethyl)carbodiimide metho- $p$-toluenesulfonate (morpho-CDI), cat. $\mathrm{CuCl}_{2}, \mathrm{CH}_{3} \mathrm{CN}$, rt (100\% yield).

The generality of this synthetic sequence was next demonstrated by the preparation of the $N$-Cbz- $N$-propargyl- $\gamma$-amino vinyl sulfones 2c and 2d. According to the synthetic strategy previously developed by us, ${ }^{7}$ the epoxide precursors $\mathbf{4 c}$ and $\mathbf{4 d}$ were first obtained from transcinnamyl and from trans-crotyl alcohols, respectively, by catalytic Sharpless epoxidation, ${ }^{10}$ regioselective opening with azide anion, ${ }^{11}$ and Mitsunobu cyclization ${ }^{6 a}$ (Scheme 5). 


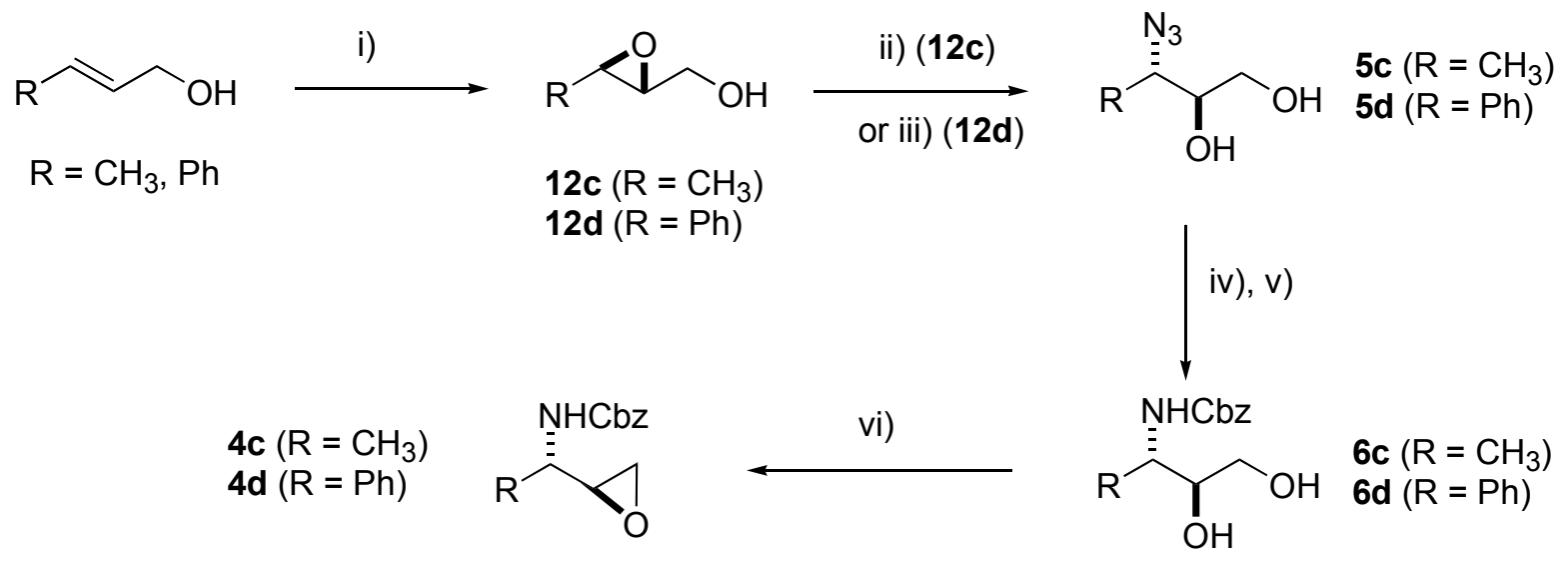

Scheme 5. i) cat. $\mathrm{Ti}\left(\mathrm{O}^{i} \mathrm{Pr}\right)_{4}$, cat. D-(-)-DIPT, TBHP, $\mathrm{CH}_{2} \mathrm{Cl}_{2},-20^{\circ} \mathrm{C}$ (12c: $53 \%$ yield, 93\% ee; 12d: $84 \%$ yield, $>98 \%$ ee); ii) $\mathrm{Ti}\left(\mathrm{O}^{i} \mathrm{Pr}\right)_{2}\left(\mathrm{~N}_{3}\right)_{2}$, toluene, $75^{\circ} \mathrm{C}\left(75 \%\right.$ yield); iii) $\mathrm{LiClO}_{4}, \mathrm{NaN}_{3}$, acetonitrile, $65^{\circ} \mathrm{C}$ (94\% yield); iv) cat. $10 \% \mathrm{Pd} / \mathrm{C}, \mathrm{H}_{2}, \mathrm{MeOH}$, rt; v) $\mathrm{PhCH}_{2} \mathrm{OCOCl}_{2} \mathrm{Et}_{3} \mathrm{~N}, 4-$ DMAP, THF, rt (6c: 55\% yield; 6d: 53\% yield, three steps); vi) $\mathrm{Ph}_{3} \mathrm{P}, \mathrm{DIAD}, \mathrm{CH}_{2} \mathrm{Cl}_{2}$, reflux (4c: $72 \%$ yield; 4d: $93 \%$ yield).

Both $4 \mathbf{c}$ and $\mathbf{4 d}$ were then submitted to the same synthetic sequence that had allowed the efficient preparation of $\mathbf{2 b}$ from $\mathbf{4 b}$ : $N$-propargylation, oxirane ring-opening with thiophenol, oxidation to the hydroxysulfone stage and carbodiimide-mediated dehydration, ${ }^{7}$ to provide the target enynes 2c and 2d (Scheme 6).

With the highly enantiopure enynes $\mathbf{2 b}, \mathbf{2 c}$ and $\mathbf{2 d}$ in our hands, we proceeded to investigate their Pauson-Khand cyclization. This reaction has lately received much attention with regard to reaction conditions using transition metal carbonyl complexes other than dicobalt octacarbonyl. $^{1 \mathrm{c}, 12}$ Thus, a number of procedures using $\mathrm{Co},{ }^{13} \mathrm{Ti},{ }^{14} \mathrm{Zr},{ }^{15} \mathrm{Ru}^{16}{ }^{16} \mathrm{Rh}^{17}$ and $\mathrm{Ir}^{18}$ have been described. In particular, the molybdenum-promoted Pauson-Khand type cyclizations, first described by Hanaoka in $1992,{ }^{19}$ constitute a useful alternative to the standard cobalt-mediated process, ${ }^{20}$ especially in the case of the intramolecular cyclocarbonylation of allenynes. ${ }^{21}$ To the best of our knowledge, however, the stereoselectivity of molybdenum-Pauson-Khand type reactions has been scarcely addressed in the literature. A few years ago, we demonstrated that an unprecedented endo-selective and regioselective intermolecular cycloaddition takes place when heterobimetallic (Mo-Co) complexes derived from $\mathrm{N}$-(2-alkynoyl)oxazolidinones or sultams are heated in the presence of norbornadiene; ${ }^{22}$ more recently, Adrio and Carretero have studied the $\mathrm{Mo}(\mathrm{CO})_{3}(\mathrm{DMF})_{3}$-mediated intramolecular reaction of electron-deficient alkenes, ${ }^{23}$ and they have found that in the case of $\gamma$-hydroxy enynes the Pauson-Khand cyclization occurs with a relatively high endo-stereoselectivity, while the reaction of the bulky triisopropylsilyloxy derivatives is always exo-selective. In this last type of substrates, the cobalt-promoted cyclization is predominantly endo-selective. ${ }^{24}$ Bearing these considerations in mind, three different experimental protocols were used for the reaction: 


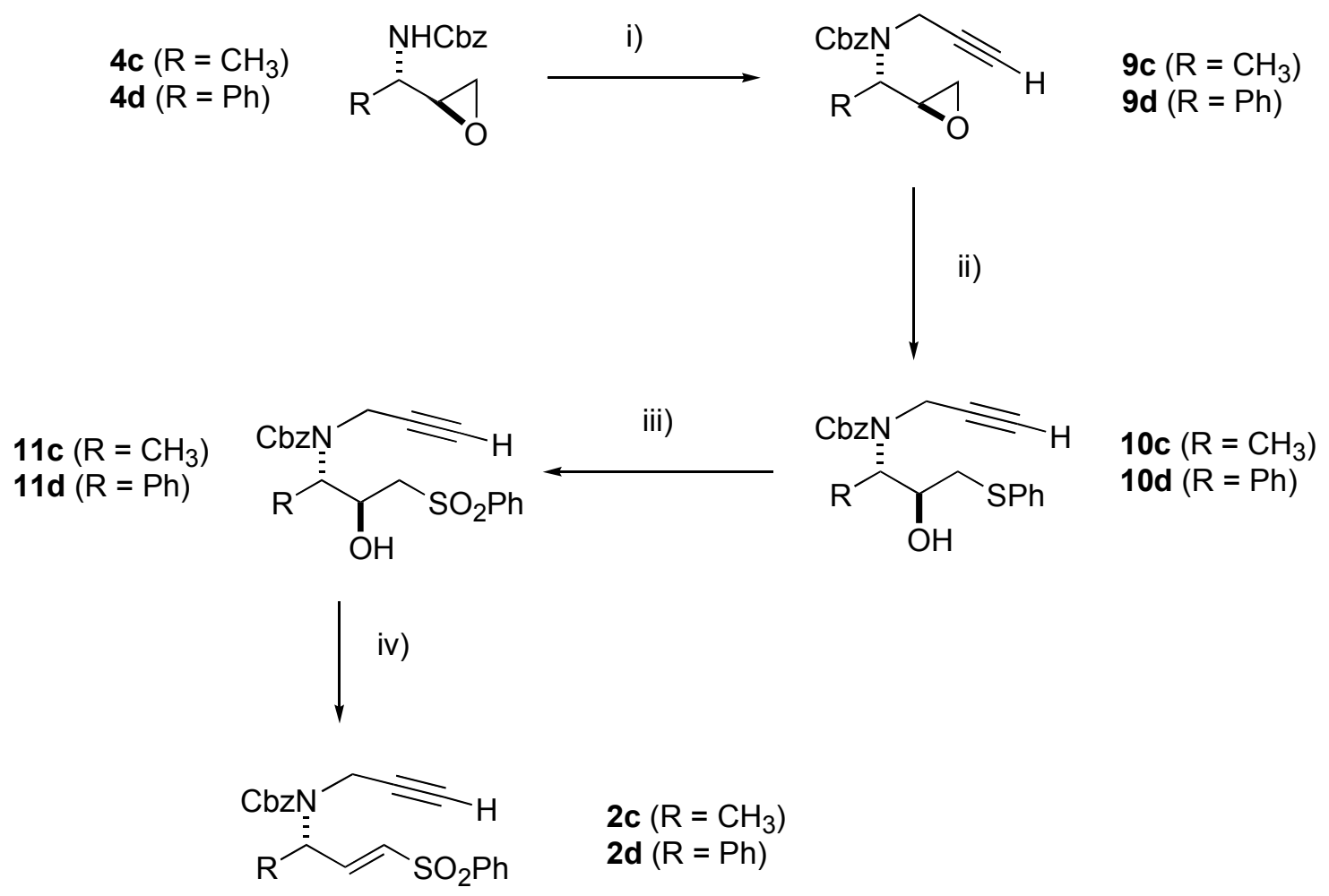

Scheme 6. i) $\mathrm{NaH}$, propargyl bromide, THF-HMPA, $\Delta$ (9c: 54\% yield; 9d: $72 \%$ yield); ii) $\mathrm{PhSH}$, $\mathrm{Et}_{3} \mathrm{~N}, \mathrm{MeOH}$, reflux (10c: 94\% yield; 10d: 91\% yield); iii) $m$-CPBA, $\mathrm{CH}_{2} \mathrm{Cl}_{2}$, rt (11c: 100\% yield; 11d: 91\% yield); iv) $N$-cyclohexyl-( $N$ '-morpholinoethyl)carbodiimide metho- $p$ toluenesulfonate (morpho-CDI), cat. $\mathrm{CuCl}_{2}, \mathrm{CH}_{3} \mathrm{CN}$, rt (2c: $98 \%$ yield; 2d: $100 \%$ yield).

Conditions A: In situ formation of the alkyne-dicobalt hexacarbonyl complex by treatment of the enyne with a slight excess of dicobalt octacarbonyl in toluene, followed by mild $\left(60^{\circ} \mathrm{C}\right)$ thermal decomposition. ${ }^{25}$

Conditions B: In situ formation of the alkyne-dicobalt hexacarbonyl complex by treatment of the enyne with a slight excess of dicobalt octacarbonyl in toluene, followed by tertiary amine $\mathrm{N}$-oxide (7.7 molar equivs.) oxidative decomposition. ${ }^{26}$

Conditions C: Molybdenum hexacarbonyl-promoted cycloaddition, according to the procedure of Jeong et al. (1.2 molar equivs. $\mathrm{Mo}(\mathrm{CO})_{6}, 5$ molar equivs. $\mathrm{DMSO}$, toluene, $\left.100^{\circ} \mathrm{C}\right) .{ }^{20}$

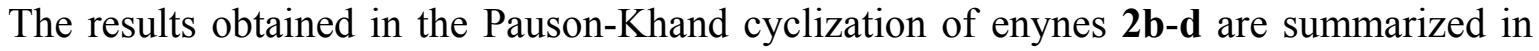
Table 1. 
Table 1. Pauson-Khand cyclization of enynes $\mathbf{2 b - d}$
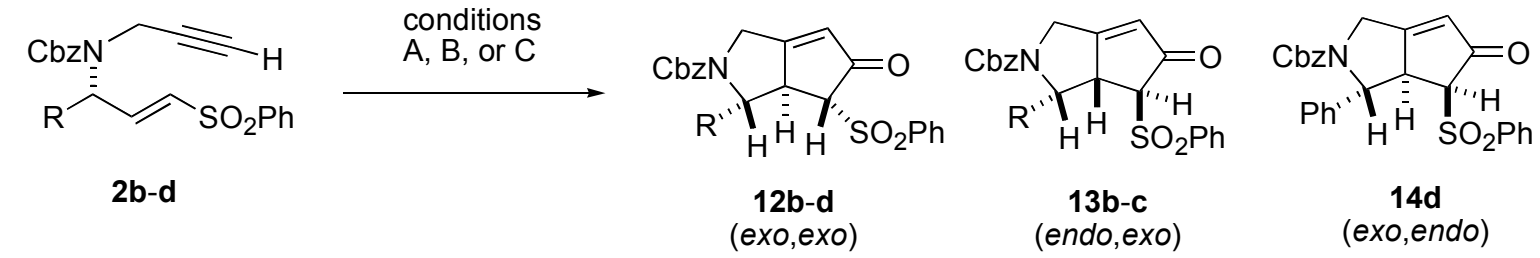

\begin{tabular}{ccccc}
\hline entry & enyne & conditions $^{\mathrm{a}}$ & global yield $(\%)^{\mathrm{b}}$ & products $(\mathrm{dr})$ \\
\hline 1 & $\mathbf{2 b}$ & $\mathrm{A}$ & 41 & $\mathbf{1 2 b}, \mathbf{1 3 b}(65: 35)$ \\
& $\left(\mathrm{R}=\left(\mathrm{CH}_{2}\right)_{3}\right.$ OTBDPS $)$ & & & \\
2 & $\mathbf{2 b}$ & $\mathrm{B}(\mathrm{NMO})$ & 22 & $\mathbf{1 2 b}$ \\
3 & $\mathbf{2 b}$ & $\mathrm{B}(\mathrm{TMANO})$ & 24 & $\mathbf{1 2 b}$ \\
4 & $\mathbf{2 b}$ & $\mathrm{C}$ & 70 & $\mathbf{1 2 b}, \mathbf{1 3 b}(20: 80)$ \\
5 & $\mathbf{2 c}\left(\mathrm{R}=\mathrm{CH}_{3}\right)$ & $\mathrm{A}$ & 12 & $\mathbf{1 2 c}, \mathbf{1 3 c}(50: 50)$ \\
6 & $\mathbf{2 c}$ & $\mathrm{B}(\mathrm{NMO})$ & 0 & - \\
7 & $\mathbf{2 c}$ & $\mathrm{C}$ & 77 & $\mathbf{1 2 c}, \mathbf{1 3 c}(33: 67)$ \\
8 & $\mathbf{2 d}(\mathrm{R}=\mathrm{Ph})$ & $\mathrm{A}$ & 46 & $\mathbf{1 2 d}, \mathbf{1 4 d}(58: 42)$ \\
9 & $\mathbf{2 d}$ & $\mathrm{B}(\mathrm{TMANO})$ & 16 & $\mathbf{1 2 d}, \mathbf{1 4 d}(50: 50)$ \\
10 & $\mathbf{2 d}$ & $\mathrm{C}$ & 65 & $\mathbf{1 2 d}$ \\
\hline
\end{tabular}

${ }^{\mathrm{a}}$ See text, ${ }^{\mathrm{b}}$ After chromatographic purification.

The behaviour of substrate $\mathbf{2 b}$ was investigated on the first place (entries 1-4 in Table 1). When the reaction was performed under thermal, Co-promoted conditions A, a 65:35 mixture (separable by column chromatography) of two diastereomeric Pauson-Khand adducts $\mathbf{1 2 b}$ and 13b was obtained in a global $41 \%$ yield (entry 1). A careful NMR spectroscopic analysis of the two compounds readily established that the major one $\mathbf{1 2 b}$ had both the side alkyl chain and the phenylsulfonyl group in the exo (or convex) face of the 3-azabicyciclo[3.3.0]oct-5-ene-7-one framework, while the minor component $\mathbf{1 3 b}$ had the opposite stereochemistry at $\mathrm{C}_{2}$ (alkyl chain in the endo or concave face). Diagnostic signals for the stereochemical assignment were the different chemical shifts for $\mathrm{H}_{2}(3.71 \mathrm{ppm}$ in $\mathbf{1 2 b}$ and $4.39 \mathrm{ppm}$ in $\mathbf{1 3 b})$, the presence of NOE between $\mathrm{H}_{2}$ and $\mathrm{H}_{8}$ in the case of $\mathbf{1 2 b}$, and a $4.2 \mathrm{~Hz}$ coupling constant between $\mathrm{H}_{1}$ and $\mathrm{H}_{2}$ (indicative of a cis relationship) in $\mathbf{1 3 b}$. Moreover, both compounds had very similar chemical shift values for $\mathrm{H}_{8}$ (3.84-3.85 ppm); see Figure 1 for the numbering of compounds 12-14.

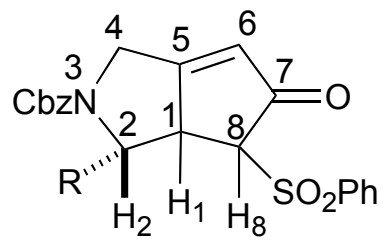

Figure 1. Atom numbering for Pauson-Khand cycloadducts 12b-d, 13b-c, and 14d 
When the cyclization was initiated by tertiary amine $N$-oxide decomposition of the intermediate alkyne-dicobalt hexacarbonyl complex (conditions B, entries 2 and 3 in Table 1), the exo,exo adduct $\mathbf{1 2 b}$ was the sole compound isolated, albeit in low yield. Interestingly enough, when molybdenum hexacarbonyl (conditions $\mathrm{C}$, entry 4 in Table 1) the endo,exo adduct $\mathbf{1 3 b}$ was the predominant one (1:4 exo,exo/endo,exo mixture, 70\% global yield). This contrasts the results of Adrio and Carretero, which pointed towards a high exo-selectivity for molybdenum-promoted Pauson-Khand intramolecular cycloadditions. ${ }^{23}$

A similar behaviour was observed for the methyl-substituted enyne 2c (entries 5-7 in Table 1). In this case, the cobalt-mediated cycloaddition was very inefficient, either under thermal (entry 5) or $\mathrm{N}$-oxide-mediated conditions (entry 6); on the other hand, the molybdenumpromoted reaction again resulted in the predominant formation of the endo,exo isomer 13c, in good global yield (entry 7). ${ }^{27}$ The stereochemistry of the adducts $12 \mathrm{c}$ and $13 \mathrm{c}$ was also established by NMR spectroscopy, and is consistent with the following observations: chemical shifts of the $\mathrm{H}_{2}$ (3.63 ppm for 12c, $4.37 \mathrm{ppm}$ for 13c) and of the $\mathrm{H}_{8}(3.87 \mathrm{ppm}$ for $12 \mathrm{c}, 3.84 \mathrm{ppm}$ for 13c) protons, and NOE enhancements (12c: NOE between $\mathrm{H}_{2}$ and $\mathrm{H}_{8}$, and between the $\mathrm{CH}_{3}$ group and $\mathrm{H}_{1} ;$ 13c: $\mathrm{NOE}$ between the $\mathrm{CH}_{3}$ and $\mathrm{H}_{8}$ ).

When we performed the cyclization of the phenyl-substituted enyne $\mathbf{2 d}$, we found that under conditions $\mathrm{C}$ (entry 10), a single product was obtained in good yield. The diagnostic $J_{\mathrm{H} 1, \mathrm{H} 2}$ value of $8.8 \mathrm{~Hz}$ (indicative of a trans stereochemistry), together with the observation of NOE between $\mathrm{H}_{2}$ and $\mathrm{H}_{8}$, established the stereochemistry of this compound as exo,exo (12d). The cobalt-mediated, thermal reaction (conditions A, entry 8) afforded a 58:42 mixture of the same compound with a diastereisomer $\mathbf{1 4 d}$ in $46 \%$ global yield. The stereochemistry of $14 \mathbf{d}$ was tentatively assigned as exo,endo, according to the $8.8 \mathrm{~Hz}$ value of the coupling constant between $\mathrm{H}_{1}$ and $\mathrm{H}_{2}$, and to the lack of NOE between $\mathrm{H}_{2}$ and $\mathrm{H}_{8}$. Also consistent with this assignment is the fact that the chemical shift values for $\mathrm{H}_{8}$ in the two isomers were no longer similar (4.00 ppm for $\mathbf{1 2 d}$ and $3.20 \mathrm{ppm}$ for $\mathbf{1 4 d}$ ). The formation of $\mathbf{1 4 d}$ could be possibly due to intermediate $\pi$ allyl-Co complexes. ${ }^{28}$

In summary, we have shown that $N$-propargyl- $\gamma$-amino vinyl sulfones, readily available in highly enantiopure fashion, are good substrates for the intramolecular Pauson-Khand reaction. The stereochemical outcome of this metal-promoted cyclization appears to be controlled not only by the substrate structure, but also by the metal carbonyl used in the reaction. While at present the mechanism and the stereochemical outcome of the standard, cobalt-mediated Pauson-Khand reactions are reasonably well understood, ${ }^{29}$ our results suggest that further work in the area of molybdenum-promoted cyclocarbonylation of enynes is needed.

\section{Experimental Section}

General Procedures. Melting points were determined in an open capillary tube and are uncorrected. Optical rotations were measured at room temperature $\left(23{ }^{\circ} \mathrm{C}\right)$; concentrations are 
given in $\mathrm{g} 100 \mathrm{ml}^{-1}$. Infrared spectra were recorded in a Fourier transform mode, using the $\mathrm{NaCl}$ film technique. Unless otherwise stated, NMR spectra were recorded in $\mathrm{CDCl}_{3}$ solution. Chemical shifts are given in ppm and referenced to TMS or $\mathrm{CHCl}_{3}$. Carbon multiplicities were established by DEPT experiments. Elemental analyses were performed by the "Servicios Xerais de Apoio á Investigación, Universidade da Coruña". MS spectra were performed at the "Servei de Espectrometria de Masses de la Universitat de Barcelona", using chemical ionization (CI) with ammonia or methane, electrospray ionization (ESI) or fast atom bombardment (FAB) techniques. Exact mass measurements (HRMS) were performed by the "Unidad de Espectrometría de Masas de la Universidad de Santiago de Compostela". Reactions were generally run in flame- or oven-dried glassware under a $\mathrm{N}_{2}$ atmosphere, with solvents dried by routine procedures. Commercially available reagents were used as received.

3-(tert-Butoxycarbonylamino)-6-(tert-butyldiphenylsilyloxy)hexa-1,3-diene (3a). To a cold $\left(0^{\circ} \mathrm{C}\right)$, stirred suspension of sodium hydride $(6 \mathrm{mg}, 0.2 \mathrm{mmol})$ in anhydrous tetrahydrofuran $(1$ $\mathrm{ml})$, a solution of the $\gamma$-amino vinyl sulfone $1 \mathbf{a}^{7}(50 \mathrm{mg}, 0.08 \mathrm{mmol})$ in tetrahydrofuran $(1 \mathrm{ml})$ was added with the aid of a syringe, and stirring was maintained for $1 \mathrm{~h}$. At this point, propargyl bromide ( $27 \mu \mathrm{l}, 0.25 \mathrm{mmol}$ ) was added in one portion. The reaction was monitored by TLC. When no starting product remained ( $20 \mathrm{~h}$ stirring at room temperature), a saturated brine solution $(2 \mathrm{ml})$ was added and stirring maintained for $5 \mathrm{~min}$. The reaction mixture was extracted with diethyl ether $(3 \times 2 \mathrm{ml})$; the organic extracts were dried over magnesium sulfate and the solvents removed at reduced pressure. Column chromatography of the crude product (silica gel, hexaneethyl acetate mixtures as eluents) afforded $25 \mathrm{mg}$ (66\% yield) of the diene 3a. ${ }^{1} \mathrm{H}$ NMR (400 $\left.\mathrm{MHz}, \mathrm{CDCl}_{3}\right),: \delta=0.98(\mathrm{~s}, 9 \mathrm{H}), 1.45(\mathrm{~s}, 9 \mathrm{H}), 2.32(\mathrm{~m}, 2 \mathrm{H}), 3.62(\mathrm{t}, J=6.0 \mathrm{~Hz}, 2 \mathrm{H}), 5.06(\mathrm{~d}, J=$ $10.4 \mathrm{~Hz}, 1 \mathrm{H}), 5.25(\mathrm{~d}, J=17.2 \mathrm{~Hz}, 1 \mathrm{H}), 5.49(\mathrm{t}, J=7.6 \mathrm{~Hz}, 1 \mathrm{H}), 6.33$, dd, $J_{1}=17.2 \mathrm{~Hz}, J_{2}=$ $10.4 \mathrm{~Hz}, 1 \mathrm{H}), 7.37-7.43(\mathrm{~m}, 6 \mathrm{H}), 7.64-7.67(\mathrm{~m}, 4 \mathrm{H}) \mathrm{ppm} .{ }^{13} \mathrm{C} \mathrm{NMR}\left(100 \mathrm{MHz}, \mathrm{CDCl}_{3}\right): \delta=19.4$ (C), $26.9\left(\mathrm{CH}_{3}\right), 28.3\left(\mathrm{CH}_{3}\right), 30.7\left(\mathrm{CH}_{2}\right), 63.6\left(\mathrm{CH}_{2}\right), 79.8(\mathrm{C}), 110.0(\mathrm{CH}), 112.5\left(\mathrm{CH}_{2}\right), 127.6$ $(\mathrm{CH}), 129.6(\mathrm{CH}), 133.4(\mathrm{C}), 131.5(\mathrm{CH}), 135.5(\mathrm{CH}), 155.9(\mathrm{C}) \mathrm{ppm} . \mathrm{MS}:\left(\mathrm{FAB}^{+}\right), m / e=474$ $\left([\mathrm{M}+23]^{+}, 10 \%\right), 452\left([\mathrm{M}+1]^{+}, 10 \%\right), 352\left([\mathrm{M}-99]^{+}, 65 \%\right)$.

\section{(3S,E)-3-[(2-Propynyl)amino)]-6-(tert-butyldiphenylsilyloxy)-1-phenylsulfonyl-1-hexene}

(2'a). To a stirred solution of $\mathbf{1 a}^{7}(100 \mathrm{mg}, 0.17 \mathrm{mmol})$ in dry dichloromethane $(2 \mathrm{ml})$ trifluoroacetic acid $(0.50 \mathrm{ml}, 6.5 \mathrm{mmol})$ was added dropwise. After stirring for $1 \mathrm{~h}$ at $\mathrm{rt}$ (TLC monitoring), the solvent was removed under vacuum. The residue was mixed with $\mathrm{K}_{2} \mathrm{CO}_{3}$ (50 $\mathrm{mg}, 0.36 \mathrm{mmol})$ and dissolved with anhydrous DMF (2 ml). To the resulting solution propargyl bromide $(25 \mu \mathrm{l}, 0.23 \mathrm{mmol})$ was added in one portion. The reaction flask was stirred at $\mathrm{rt}$, protected from sunlight, during $24 \mathrm{~h}$. The resulting mixture was dilluted with $\mathrm{CH}_{2} \mathrm{Cl}_{2}(5 \mathrm{ml})$ and washed with $\mathrm{H}_{2} \mathrm{O}(5 \mathrm{ml})$. The aqueous phase was separated and washed with $\mathrm{CH}_{2} \mathrm{Cl}_{2}(3 \times 5 \mathrm{ml})$. The combined organic phases were dried over anhydrous $\mathrm{MgSO}_{4}$ and the solvents were removed under vacuum. Column chromatography of the crude product (silica gel, hexane-ethyl acetate mixtures as eluents) afforded $54 \mathrm{mg}(60 \%$ yield) of the enyne 2'a, as a colorless oil, and $15 \mathrm{mg}$ of dipropargylated product. $[\alpha]_{\mathrm{D}}{ }^{23}=+11.6\left(c=0.75, \mathrm{CHCl}_{3}\right)$. IR $\left(\mathrm{NaCl}\right.$ film): $v_{\max }=2931,1447$, 1428, 1308, 1148, 1111, 1086, 704, $687 \mathrm{~cm}^{-1} .{ }^{1} \mathrm{H}-\mathrm{NMR}\left(200 \mathrm{MHz}, \mathrm{CDCl}_{3}\right): \delta=1.03(\mathrm{~s}, 9 \mathrm{H})$, 
1.51-1.64 (m, 4H), $2.14(\mathrm{t}, J=2.4 \mathrm{~Hz}, 1 \mathrm{H}), 3.19\left(\mathrm{dd}, J_{l}=17.2 \mathrm{~Hz}, J_{2}=2.4 \mathrm{~Hz}, 1 \mathrm{H}\right), 3.19\left(\mathrm{dd}, J_{1}\right.$ $\left.=17.2 \mathrm{~Hz}, J_{2}=2.4 \mathrm{~Hz}, 1 \mathrm{H}\right), 3.49(\mathrm{~m}, 1 \mathrm{H}), 3.63(\mathrm{t}, J=6.0 \mathrm{~Hz}, 2 \mathrm{H}),, 6.50\left(\mathrm{dd}, J_{1}=15.2 \mathrm{~Hz}, J_{2}=\right.$ $0.8 \mathrm{~Hz}, 1 \mathrm{H}), 6.78\left(\mathrm{dd}, J_{1}=15.2 \mathrm{~Hz}, J_{2}=7.2 \mathrm{~Hz}, 1 \mathrm{H}\right), 7.35-7.64(\mathrm{~m}, \mathrm{NH}+13 \mathrm{H}), 7.87-7.89(\mathrm{~m}$, 2H) ppm. ${ }^{13} \mathrm{C}-\mathrm{NMR}\left(100 \mathrm{MHz}, \mathrm{CDCl}_{3}\right): \delta=19.2(\mathrm{C}), 26.9\left(\mathrm{CH}_{3}\right), 28.4\left(\mathrm{CH}_{2}\right), 31.0\left(\mathrm{CH}_{2}\right), 36.0$ $\left(\mathrm{CH}_{2}\right), 57.0(\mathrm{CH}), 63.3\left(\mathrm{CH}_{2},\right), 72.0(\mathrm{CH}), 81.5(\mathrm{C}), 127.6(\mathrm{CH}), 127.7(\mathrm{CH}), 129.3(\mathrm{CH}), 129.4$ $(\mathrm{CH}), 129.7(\mathrm{CH}), 131.6(\mathrm{CH}), 133.4(\mathrm{CH}), 133.7(\mathrm{C}), 135.6(\mathrm{CH}), 140.5(\mathrm{C}), 147.6(\mathrm{CH}) \mathrm{ppm}$. $\mathrm{MS}\left(\mathrm{FAB}^{+}, \mathrm{NBA}\right) m / e: 532\left([\mathrm{M}+1]^{+}, 30 \%\right)$.

(2S,3S)-3-(Benzyloxycarbonylamino-6-(tert-butyldiphenylsilyloxy)-1,2-hexanediol (6). A solution of azidodiol $5^{7}(1.65 \mathrm{~g}, 4.0 \mathrm{mmol})$ in anhydrous $\mathrm{MeOH}(40 \mathrm{ml})$ was stirred at room temperature under hydrogen (balloon) in the presence of $10 \% \mathrm{Pd} / \mathrm{C}(165 \mathrm{mg})$ during $16 \mathrm{~h}$ (TLC monitoring). After filtration through Celite ${ }^{\circledR}$ and removal of the solvent $(2 S, 3 S)$-3-amino-6-(tertbutyldiphenylsilyloxy)-1,2-hexanediol (1.65 g, quant. yield) was obtained. ${ }^{1} \mathrm{H}-\mathrm{NMR}(400 \mathrm{MHz}$, $\left.\mathrm{CDCl}_{3}\right): \delta 1.05(\mathrm{~s}, 9 \mathrm{H}), 1.52-1.76(\mathrm{~m}, 4 \mathrm{H}), 2.46($ br s, $2 \mathrm{H}, \mathrm{OH}), 3.01(\mathrm{~m}, 1 \mathrm{H}), 3.48(\mathrm{~m}, 1 \mathrm{H}), 3.69$ $(\mathrm{m}, 4 \mathrm{H}), 7.38-7.41(\mathrm{~m}, 6 \mathrm{H}), 7.63-7.68(\mathrm{~m}, 4 \mathrm{H}) \mathrm{ppm}$.

A solution of this crude aminodiol $(2.5 \mathrm{~g}, 6.2 \mathrm{mmol})$ in anhydrous tetrahydrofuran $(20 \mathrm{ml})$ was treated with triethylamine $(1 \mathrm{ml}, 7.5 \mathrm{mmol}), 4-D M A P(75 \mathrm{mg}, 0.6 \mathrm{mmol})$ and benzyl chloroformate $(1.1 \mathrm{ml}, 7.5 \mathrm{mmol})$ for $4 \mathrm{~h}$ at room temperature (TLC monitoring). The resulting solution was washed with brine $(20 \mathrm{ml})$, and the aqueous phase was extracted with diethyl ether $(3 \times 10 \mathrm{ml})$. The combined organic phases were dried over anhydrous $\mathrm{MgSO}_{4}$ and the solvents were removed under vacuum. Column chromatography of the crude product $(2.5 \% \mathrm{v} / \mathrm{v}$ triethylamine-pretreated silica gel, hexane-ethyl acetate mixtures as eluents) afforded $2.1 \mathrm{~g}(65 \%$ yield) of the title compound, as a colorless oil. $[\alpha]_{\mathrm{D}}{ }^{25}=-3.8\left(c=1.0, \mathrm{CHCl}_{3}\right)$. IR (film NaCl): $v_{\max }=3400,2933,2860,1700,1522,1457,1428,1245,1111,741,702 \mathrm{~cm}^{-1} .{ }^{1} \mathrm{H}-\mathrm{NMR}(400$ $\mathrm{MHz}_{\mathrm{CDCl}}$ ): $\delta 1.04(\mathrm{~s}, 9 \mathrm{H}), 1.52-1.76(\mathrm{~m}, 4 \mathrm{H}), 2.64$ (br s, 1H, OH), 3.07 (br s, $\left.1 \mathrm{H}, \mathrm{OH}\right), 3.38$ $(\mathrm{m}, 1 \mathrm{H}), 3.66(\mathrm{~m}, 5 \mathrm{H}), 5.02(\mathrm{br} \mathrm{d}, 1 \mathrm{H}, \mathrm{NH}), 5.12(\mathrm{~m}, 2 \mathrm{H}), 7.34-7.39(\mathrm{~m}, 9 \mathrm{H}), 7.62-7.65(\mathrm{~m}, 6 \mathrm{H})$ ppm. ${ }^{13} \mathrm{C}-\mathrm{NMR}\left(100 \mathrm{MHz}, \mathrm{CDCl}_{3}\right): \delta 19.2(\mathrm{C}), 26.9\left(\mathrm{CH}_{3}\right), 27.4\left(\mathrm{CH}_{2}\right), 28.8(\mathrm{CH}), 53.0(\mathrm{CH})$, $62.9\left(\mathrm{CH}_{2}\right), 63.4\left(\mathrm{CH}_{2}\right), 67.2\left(\mathrm{CH}_{2}\right), 74.2(\mathrm{CH}), 127.7(\mathrm{CH}), 128.1(\mathrm{CH}), 128.3(\mathrm{CH}), 128.6$ $(\mathrm{CH}), 129.6(\mathrm{CH}), 133.7(\mathrm{C}), 135.5(\mathrm{CH}), 136.1(\mathrm{C}), 157.7$ (C) ppm. MS (ESI) m/e: 523 $\left([\mathrm{M}+1]^{+}, 25 \%\right), 401$ ([M-121] $\left.]^{+}, 100 \%\right)$. HRMS (ESI) $\mathrm{C}_{30} \mathrm{H}_{39} \mathrm{NNaO}_{5} \mathrm{Si}(\mathrm{M}+\mathrm{Na}): 544.2490$ calcd., 544.2471 found.

(S)-2-[(S)-1-(Benzyloxycarbonylamino)-4-(tert-butyldiphenylsilyloxy)butyl]oxirane (4b). To a solution of diol $5(5.0 \mathrm{~g}, 9.6 \mathrm{mmol})$ and of triphenylphosphine $(2.7 \mathrm{~g}, 10.3 \mathrm{mmol})$ in anhydrous chloroform $(40 \mathrm{ml})$ a solution of diisopropylazodicarboxylate $(2.0 \mathrm{ml}, 10.3 \mathrm{mmol})$ in anhydrous chloroform $(10 \mathrm{ml})$ was added dropwise. The resulting mixture was heated to reflux until total disappearance of the starting diol (14 h, TLC monitoring). The solvent was removed at reduced pressure and the residue was purified by column chromatography (silica gel, hexane-ethyl acetate mixtures as eluents) to afford $4.3 \mathrm{~g} \quad(89 \%$ yield $)$ of $(S)-2-[(S)-1$ (benzyloxycarbonylamino)-4-(tert-butyldiphenylsilyloxy)butyl]oxirane $\mathbf{4 b}$ as a colorless oil. $[\alpha]_{\mathrm{D}}{ }^{23}=-6.9\left(c=1.0, \mathrm{CHCl}_{3}\right) . \mathrm{IR}(\mathrm{NaCl}$ film $): v_{\max }=3330,3070,2931,2858,1710,1428,1239$, 1111, 739, $702 \mathrm{~cm}^{-1} .{ }^{1} \mathrm{H}-\mathrm{NMR}\left(400 \mathrm{MHz}, \mathrm{CDCl}_{3}\right): \delta 1.04(\mathrm{~s}, 9 \mathrm{H}), 1.52-1.74(\mathrm{~m}, 4 \mathrm{H}), 2.75(\mathrm{~m}$, 
2H), $2.88(\mathrm{~m}, 1 \mathrm{H}), 3.48(\mathrm{~m}, 1 \mathrm{H}), 3.67(\mathrm{t}, J=5.4 \mathrm{~Hz}, 2 \mathrm{H}), 4.79$ (br d, 1H, NH), $5.09(\mathrm{~s}, 2 \mathrm{H}), 7.34-$ $7.40(\mathrm{~m}, 9 \mathrm{H}), 7.63-7.67(\mathrm{~m}, 6 \mathrm{H}) \mathrm{ppm} .{ }^{13} \mathrm{C}-\mathrm{NMR}\left(100 \mathrm{MHz}, \mathrm{CDCl}_{3}\right): \delta 19.2(\mathrm{C}), 26.9\left(\mathrm{CH}_{3}\right), 28.1$ $\left(\mathrm{CH}_{2}\right), 28.5\left(\mathrm{CH}_{2}\right), 46.1\left(\mathrm{CH}_{2}\right), 52.4(\mathrm{CH}), 53.9(\mathrm{CH}), 63.3\left(\mathrm{CH}_{2}\right), 66.8\left(\mathrm{CH}_{2}\right), 127.6(\mathrm{CH}), 128.0$ $(\mathrm{CH}), 128.1(\mathrm{CH}), 128.5(\mathrm{CH}), 129.5(\mathrm{CH}), 133.80(\mathrm{C}), 133.85(\mathrm{C}), 135.4(\mathrm{CH}), 156.0(\mathrm{C}) \mathrm{ppm}$. MS (ESI) m/e: 505 ([M+1] $]^{+}, 100 \%$ ). HRMS (ESI) $\mathrm{C}_{30} \mathrm{H}_{37} \mathrm{NNaO}_{4} \mathrm{Si}(\mathrm{M}+\mathrm{Na}): 526.2384$ calcd., 526.2371 found.

(2S,3S)-3-(Benzyloxycarbonylamino)-6-(tert-butyldiphenylsilyloxy)-1-phenylthio-2-hexanol (7). To a solution of oxirane $4 \mathbf{b}(0.90 \mathrm{~g}, 1.8 \mathrm{mmol})$ in anhydrous methanol $(18 \mathrm{ml})$ triethylamine (254 $\mu \mathrm{l}, 1.8 \mathrm{mmol})$ and thiophenol $(184 \mu \mathrm{l}, 1.8 \mathrm{mmol})$ were added sequentially. The reaction mixture was heated to reflux until total disappearance of the starting oxirane $(2 \mathrm{~h}$, TLC monitoring). The solvent was removed at reduced pressure and the residue was purified by column chromatography $(2.5 \% \mathrm{v} / \mathrm{v}$ triethylamine-pretreated silica gel, hexane-ethyl acetate mixtures as eluents) to afford $1.0 \mathrm{~g}$ (91\% yield) of (2S,3S)-3-(benzyloxycarbonylamino)-6-(tertbutyldiphenylsilyloxy)-1-phenylthio-2-hexanol 7 as a colorless solid. Mp $83-84^{\circ} \mathrm{C}$. $[\alpha]_{\mathrm{D}}{ }^{23}=-9.4$ $\left(c=1.0, \mathrm{CHCl}_{3}\right)$. IR ( NaCl film): $v_{\max }=3406,3072,2931,2858,1700,1515,1428,1239,1111$, 1027, 739, $702 \mathrm{~cm}^{-1} .{ }^{1} \mathrm{H}-\mathrm{NMR}\left(400 \mathrm{MHz}, \mathrm{CDCl}_{3}\right): \delta=1.04(\mathrm{~s}, 9 \mathrm{H}), 1.46-1.76(\mathrm{~m}, 4 \mathrm{H}), 2.87(\mathrm{~m}$, $\mathrm{OH}+1 \mathrm{H}), 3.17(\mathrm{~d}, J=12.8 \mathrm{~Hz}, 1 \mathrm{H}), 3.66(\mathrm{~m}, 4 \mathrm{H}), 4.96(\mathrm{br} \mathrm{d}, 1 \mathrm{H}, \mathrm{NH}), 5.08(\mathrm{~s}, 2 \mathrm{H}), 7.21-7.39$ (m, 16H), 7.63-7.67 (m, 4H) ppm. ${ }^{13} \mathrm{C}-\mathrm{NMR}\left(100 \mathrm{MHz}, \mathrm{CDCl}_{3}\right): \delta=19.4(\mathrm{C}), 26.1\left(\mathrm{CH}_{2}\right), 27.1$ $\left(\mathrm{CH}_{3}\right), 29.1\left(\mathrm{CH}_{2}\right), 39.0\left(\mathrm{CH}_{2}\right), 55.2(\mathrm{CH}), 63.7\left(\mathrm{CH}_{2}\right), 67.1\left(\mathrm{CH}_{2}\right), 72.3(\mathrm{CH}), 127.0(\mathrm{CH}), 127.9$ $(\mathrm{CH}), 128.3(\mathrm{CH}), 128.4(\mathrm{CH}), 128.8(\mathrm{CH}), 129.4(\mathrm{CH}), 129.8(\mathrm{CH}), 130.4(\mathrm{CH}), 134.0(\mathrm{C})$, $134.1(\mathrm{C}), 135.8(\mathrm{CH}), 136.6(\mathrm{C}), 156.8$ (C) ppm. MS (ESI) m/e: 615 ([M+1] $\left.]^{+}, 100 \%\right)$. HRMS (ESI) $\mathrm{C}_{36} \mathrm{H}_{43} \mathrm{NNaO}_{4} \mathrm{SSi}(\mathrm{M}+\mathrm{Na})$ : 636.2574 calcd., 636.2551 found. Anal. $\left(\mathrm{C}_{36} \mathrm{H}_{43} \mathrm{NO}_{4} \mathrm{SSi}\right)$, (calcd., found): \%C (70.43, 70.40), \%H (7.06, 7.13), \%N (2.28, 2.35), \% $\mathrm{S}(5.22,4.61)$.

\section{(2S,3S)-3-(Benzyloxycarbonylamino)-6-(tert-butyldiphenylsilyloxy)-1-phenylsulfonyl-2-}

hexanol (8). To a solution of the hydroxysulfide 7 (1.0 g, $1.6 \mathrm{mmol})$ in anhydrous dichloromethane $(30 \mathrm{ml})$ a solution of $m$-CPBA $(0.70 \mathrm{~g}, 4.1 \mathrm{mmol})$ in dry dichloromethane $(30$ $\mathrm{ml}$ ) was added dropwise. The reaction mixture was stirred at room temperature until total disappearance of the starting sulfide ( $2 \mathrm{~h}$, TLC monitoring), and cooled to $0^{\circ} \mathrm{C}$. After addition of a $10 \%$ aqueous solution of sodium sulfite $(25 \mathrm{ml})$, stirring was maintained at the same temperature for $15 \mathrm{~min}$. The organic phase was separated and washed with a saturated solution of sodium bicarbonate $(3 \times 10 \mathrm{ml})$ and with brine $(30 \mathrm{ml})$. The organic phase was dried over anhydrous $\mathrm{MgSO}_{4}$ and the solvent was removed under vacuum. Column chromatography of the crude product $(2.5 \% \mathrm{v} / \mathrm{v}$ triethylamine-pretreated silica gel, hexane-ethyl acetate mixtures as eluents) afforded $\quad 1.0 \mathrm{~g} \quad(95 \%$ yield) of (2S,3S)-3-(benzyloxycarbonylamino)-6-(tertbutyldiphenylsilyloxy)-1-phenylsulfonyl-2-hexanol 8 as a dense, colorless oil. $[\alpha]_{\mathrm{D}}{ }^{23}=-2.9(c=$ 1.0, $\mathrm{CHCl}_{3}$ ). IR ( $\mathrm{NaCl}$ film): $v_{\max }=3355,3070,2933,1702,1522,1447,1428,1306,1241$, 1146, 1111, $743 \mathrm{~cm}^{-1} .{ }^{1} \mathrm{H}-\mathrm{NMR}\left(400 \mathrm{MHz}, \mathrm{CDCl}_{3}\right): \delta=1.02(\mathrm{~s}, 9 \mathrm{H}), 1.46-1.74(\mathrm{~m}, 4 \mathrm{H}), 3.22(\mathrm{br}$ $\mathrm{d}, 2 \mathrm{H}), 3.62(\mathrm{~m}, 3 \mathrm{H}), 4.15(\mathrm{~m}, 1 \mathrm{H}), 4.96(\mathrm{br} \mathrm{d}, 1 \mathrm{H}, \mathrm{NH}), 5.05(\mathrm{~s}, 2 \mathrm{H}), 7.32-7.38(\mathrm{~m}, 10 \mathrm{H}), 7.60-$ $7.63(\mathrm{~m}, 8 \mathrm{H}), 7.89-7.93(\mathrm{~m}, 2 \mathrm{H}) \mathrm{ppm} .{ }^{13} \mathrm{C}-\mathrm{NMR}\left(100 \mathrm{MHz}, \mathrm{CDCl}_{3}\right): \delta=19.4(\mathrm{C}), 26.2\left(\mathrm{CH}_{2}\right)$, $27.1\left(\mathrm{CH}_{3}\right), 28.8\left(\mathrm{CH}_{2}\right), 55.1(\mathrm{CH}), 60.0\left(\mathrm{CH}_{2}\right), 63.5\left(\mathrm{CH}_{2}\right), 67.2\left(\mathrm{CH}_{2}\right), 68.9(\mathrm{CH}), 127.9(\mathrm{CH})$, 
$128.2(\mathrm{CH}), 128.3(\mathrm{CH}), 128.5(\mathrm{CH}), 128.8(\mathrm{CH}), 129.7(\mathrm{CH}), 129.9(\mathrm{CH}), 133.9(\mathrm{C}), 134.4$ $(\mathrm{CH}), 135.5(\mathrm{CH}), 135.8(\mathrm{C}), 139.4(\mathrm{C}), 156.4(\mathrm{C}) \mathrm{ppm}$. MS (ESI) m/e: $647\left([\mathrm{M}+1]^{+}, 100 \%\right)$. HRMS (ESI) $\mathrm{C}_{36} \mathrm{H}_{43} \mathrm{NNaO}_{6} \mathrm{SSi}(\mathrm{M}+\mathrm{Na}): 668.2473$ calcd., 668.2469 found.

\section{(3S,E)-3-(Benzyloxycarbonylamino)-6-(tert-butyldiphenylsilyloxy)-1-phenylsulfonyl-1-}

hexene (1b). To a cold $\left(0^{\circ} \mathrm{C}\right)$, stirred solution of the hydroxysulfone $8(130 \mathrm{mg}, 0.20 \mathrm{mmol})$ and of 4-DMAP (100 mg, $0.80 \mathrm{mmol})$ in anhydrous dichloromethane $(2 \mathrm{ml})$ a solution of methanesulfonyl chloride $(31 \mu \mathrm{l}, 0.40 \mathrm{mmol})$ in dry dichloromethane $(1 \mathrm{ml})$ was added dropwise. The reaction mixture was stirred at room temperature until total disappearance of the starting hydroxysulfone (7 h, TLC monitoring), diluted with dichloromethane $(2 \mathrm{ml})$, and washed with a cold $\left(0^{\circ} \mathrm{C}\right) 10 \%$ aqueous solution of $\mathrm{HCl}(5 \mathrm{ml})$ and with a saturated aqueous solution of sodium bicarbonate $(5 \mathrm{ml})$. The organic phase was dried over anhydrous $\mathrm{MgSO}_{4}$ and the solvent was removed under vacuum. Column chromatography of the crude product $(2.5 \% \mathrm{v} / \mathrm{v}$ triethylaminepretreated silica gel, hexane-ethyl acetate mixtures as eluents) afforded $94 \mathrm{mg}$ (75\% yield) of (3S,E)-3-(benzyloxycarbonylamino)-6-(tert-butyldiphenylsilyloxy)-1-phenylsulfonyl-1-hexene $\mathbf{1 b}$ as a dense, colorless oil. $[\alpha]_{\mathrm{D}}^{23}=+5.0\left(\mathrm{c}=1.0, \mathrm{CHCl}_{3}\right)$. IR $(\mathrm{NaCl}$ film $): v_{\max }=3344,3070$, 2931, 2858, 1719, 1522, 1447, 1429, 1308, 1245, 1148, $1111 \mathrm{~cm}^{-1} .{ }^{1} \mathrm{H}-\mathrm{NMR}\left(400 \mathrm{MHz}, \mathrm{CDCl}_{3}\right)$ : $\delta=1.03(\mathrm{~s}, 9 \mathrm{H}), 1.50-1.78(\mathrm{~m}, 4 \mathrm{H}), 3.63(\mathrm{t}, J=5.4 \mathrm{~Hz}, 2 \mathrm{H}), 4.42(\mathrm{br} \mathrm{m}, 1 \mathrm{H}), 4.90(\mathrm{br} \mathrm{d}, 1 \mathrm{H}$, $\mathrm{NH}), 6.38\left(\mathrm{dd}, J_{1}=15.0 \mathrm{~Hz}, J_{2}=1.4 \mathrm{~Hz}, 1 \mathrm{H}\right), 6.88\left(\mathrm{dd}, J_{l}=15.0 \mathrm{~Hz}, J_{2}=5.5 \mathrm{~Hz}, 1 \mathrm{H}\right), 7.31-7.40$ (m, 10H), 7.52-7.64 (m, 8H), 7.82-7.89 (m, 2H) ppm. ${ }^{13} \mathrm{C}-\mathrm{NMR}\left(100 \mathrm{MHz}, \mathrm{CDCl}_{3}\right): \delta=19.2$ (C), $26.9\left(\mathrm{CH}_{3}\right), 28.4\left(\mathrm{CH}_{2}\right), 30.7\left(\mathrm{CH}_{2}\right), 51.2(\mathrm{CH}), 62.9\left(\mathrm{CH}_{2}\right), 67.1\left(\mathrm{CH}_{2}\right), 127.6(\mathrm{CH}), 127.7$ $(\mathrm{CH}), 128.0(\mathrm{CH}), 128.2(\mathrm{CH}), 128.5(\mathrm{CH}), 129.2(\mathrm{CH}), 129.6(\mathrm{CH}), 130.4(\mathrm{CH}), 133.3(\mathrm{C})$, $133.4(\mathrm{CH}), 135.4(\mathrm{CH}), 140.3(\mathrm{C}), 146.0(\mathrm{CH}), 155.5(\mathrm{C}) \mathrm{ppm}$. MS (ESI) m/e: $629\left([\mathrm{M}+1]^{+}\right.$, $100 \%$ ). HRMS (ESI) $\mathrm{C}_{36} \mathrm{H}_{41} \mathrm{NNaO}_{5} \mathrm{SSi}(\mathrm{M}+\mathrm{Na}): 650.2367$ calcd., 650.2339 found.

3-(Benzyloxycarbonylamino)-6-(tert-butyldiphenylsilyloxy)hexa-1,3-diene (3b). To a cold $\left(0^{\circ} \mathrm{C}\right)$, stirred suspension of sodium hydride $(3.5 \mathrm{mg}, 0.13 \mathrm{mmol})$ in anhydrous tetrahydrofuran $(1$ $\mathrm{ml})$, a solution of the $\gamma$-amino vinyl sulfone $1 \mathbf{b}(70 \mathrm{mg}, 0.11 \mathrm{mmol})$ in dry tetrahydrofuran $(1 \mathrm{ml})$ was added with the aid of a syringe, and stirring was maintained for $1 \mathrm{~h}$. At this point, propargyl bromide ( $15 \mu \mathrm{l}, 0.14 \mathrm{mmol})$ was added in one portion. The reaction was monitored by TLC. When no starting product remained ( $1 \mathrm{~h}$ stirring at room temperature), a saturated sodium bicarbonate solution $(2 \mathrm{ml})$ was added and stirring maintained for $5 \mathrm{~min}$. The reaction mixture was extracted with dichloromethane $(3 \times 2 \mathrm{ml})$; the organic extracts were dried over magnesium sulfate and the solvents removed at reduced pressure. Column chromatography of the crude product (silica gel, hexane-ethyl acetate mixtures as eluents) afforded $20 \mathrm{mg}$ (37\% yield) of the diene 3b. ${ }^{1} \mathrm{H}$ NMR $\left(200 \mathrm{MHz}, \mathrm{CDCl}_{3}\right): \delta=1.03(\mathrm{~s}, 9 \mathrm{H}), 2.38(\mathrm{~m}, 2 \mathrm{H}), 3.64(\mathrm{t}, J=6.0 \mathrm{~Hz}, 2 \mathrm{H})$, $5.06(\mathrm{~d}, J=10.4 \mathrm{~Hz}, 1 \mathrm{H}), 5.13(\mathrm{~s}, 2 \mathrm{H}), 5.23(\mathrm{~d}, J=17.2 \mathrm{~Hz}, 1 \mathrm{H}), 5.54(\mathrm{t}, J=7.6 \mathrm{~Hz}, 1 \mathrm{H}), 6.36$ (dd, $\left.J_{I}=17.2 \mathrm{~Hz}, J_{2}=10.4 \mathrm{~Hz}, 1 \mathrm{H}\right), 7.35-7.39(\mathrm{~m}, 11 \mathrm{H}), 7.62-7.66(\mathrm{~m}, 4 \mathrm{H}) \mathrm{ppm}$.

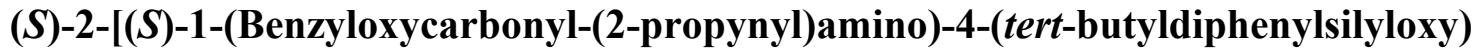

butyl]oxirane (9b). To a stirred suspension of sodium hydride $(0.20 \mathrm{~g}, 60 \%$ in paraffines, 5.0 $\mathrm{mmol})$ in anhydrous tetrahydrofuran $(15 \mathrm{ml})$ a solution of oxirane $4 \mathbf{b}(0.80 \mathrm{~g}, 1.6 \mathrm{mmol})$ in anhydrous tetrahydrofuran $(15 \mathrm{ml})$ was added dropwise, and stirring was maintained at room 
temperature for $15 \mathrm{~min}$. At this point, propargyl bromide $(1.8 \mathrm{ml}, 16 \mathrm{mmol})$ and HMPA $(14 \mathrm{ml}$, $80 \mathrm{mmol}$ ) were added sequentially with the aid of a syringe. The resulting mixture was heated to reflux for $2 \mathrm{~h}$. After cooling to room temperature, a saturated brine solution ( $15 \mathrm{ml})$ was added and stirring maintained for $5 \mathrm{~min}$. The reaction mixture was extracted with diethyl ether $(3 \times 10$ $\mathrm{ml}$ ); the organic extracts were dried over magnesium sulfate and the solvents removed at reduced pressure. Column chromatography of the crude product (silica gel, hexane-ethyl acetate mixtures as eluents) afforded $711 \mathrm{mg} \quad(82 \%$ yield $)$ of $(S)-2-[(S)-1$-(benzyloxycarbonyl-(2propynyl)amino)-4-(tert-butyldiphenylsilyloxy)butyl]oxirane $\mathbf{9 b}$ as a colorless oil. $[\alpha]_{\mathrm{D}}{ }^{23}=-9.0$ $\left(c=1.0, \mathrm{CHCl}_{3}\right) . \mathrm{IR}\left(\mathrm{NaCl}\right.$ film): $v_{\max }=3745,2931,1702,1654,1559,1540,1507,1457,1256$, 1111, $702 \mathrm{~cm}^{-1} .{ }^{1} \mathrm{H}-\mathrm{NMR}\left(400 \mathrm{MHz}, \mathrm{CDCl}_{3}\right): \delta=1.04(\mathrm{~s}, 9 \mathrm{H}), 1.46-1.82(\mathrm{~m}, 4 \mathrm{H}), 2.18(\mathrm{~s}, 1 \mathrm{H})$, 2.67 (br m, 2H), 3.18 (br m, 1H), 3.64 (br m, 2H), 3.92 (br m, 1H), 4.04 (m, 2H), 5.16 (s, 2H,), 7.32-7.42 (m, 12H), 7.62-7.66 (m, 3H) ppm. ${ }^{13} \mathrm{C}-\mathrm{NMR}\left(100 \mathrm{MHz}, \mathrm{CDCl}_{3}\right): \delta=19.4(\mathrm{C}), 27.1$ $\left(\mathrm{CH}_{3}\right), 29.1\left(\mathrm{CH}_{2}\right), 29.9\left(\mathrm{CH}_{2}\right), 34.3\left(\mathrm{CH}_{2}\right), 45.6\left(\mathrm{CH}_{2}\right), 53.6(\mathrm{CH}), 58.6(\mathrm{CH}), 63.5\left(\mathrm{CH}_{2}\right), 67.7$ $\left(\mathrm{CH}_{2}\right), 71.6(\mathrm{CH}), 80.4(\mathrm{C}), 127.6(\mathrm{CH}), 128.0(\mathrm{CH}), 128.1(\mathrm{CH}), 128.5(\mathrm{CH}), 129.5(\mathrm{CH}), 133.8$ (C), $135.5(\mathrm{CH}), 155.6(\mathrm{C})$ ppm. MS (FAB $\left.{ }^{+}, \mathrm{NBA}\right) m / e: 564\left([\mathrm{M}+23]^{+}, 100 \%\right), 542\left([\mathrm{M}+1]^{+}\right.$, 50\%). HRMS (FAB $\left.{ }^{+}\right) \mathrm{C}_{33} \mathrm{H}_{40} \mathrm{NO}_{4} \mathrm{Si}(\mathrm{M}+1)$ : 542.2727 calcd., 542.2708 found.

\section{(2S,3S)-3-(Benzyloxycarbonyl-(2-propynyl)amino)-6-(tert-butyldiphenylsilyloxy)-1-}

phenylthio-2-hexanol (10b). To a solution of oxirane 9b (1.25 g, $2.3 \mathrm{mmol})$ in anhydrous methanol $(25 \mathrm{ml})$, triethylamine $(350 \mu \mathrm{l}, 2.5 \mathrm{mmol})$ and thiophenol $(250 \mu \mathrm{l}, 2.5 \mathrm{mmol})$ were added sequentially. The reaction mixture was heated to reflux until total disappearance of the starting oxirane $(1.5 \mathrm{~h}$, TLC monitoring). The solvent was removed at reduced pressure and the residue was purified by column chromatography $(2.5 \% \mathrm{v} / \mathrm{v}$ triethylamine-pretreated silica gel, hexane-ethyl acetate mixtures as eluents) to afford $1.40 \mathrm{~g}$ (93\% yield) of (2S,3S)-3(benzyloxycarbonyl-(2-propynyl)amino)-6-(tert-butyldiphenylsilyloxy)-1-phenylthio-2-hexanol as a colorless oil. $[\alpha]_{\mathrm{D}}^{23}=-5.2\left(c=1.0, \mathrm{CHCl}_{3}\right)$. IR $(\mathrm{NaCl}$ film $): v_{\max }=3295,3071,2930,2858$, 1700, 1427, 1256, 1111, 739, $702 \mathrm{~cm}^{-1} .{ }^{1} \mathrm{H}-\mathrm{NMR}\left(400 \mathrm{MHz}, \mathrm{CDCl}_{3}\right): \delta=1.03(\mathrm{~s}, 9 \mathrm{H}), 1.46(\mathrm{~m}$, 2H), $1.83(\mathrm{~m}, 2 \mathrm{H}), 2.00(\mathrm{~s}, 1 \mathrm{H}), 2.87(\mathrm{~m}, \mathrm{OH}+1 \mathrm{H}), 3.17(\mathrm{~m}, 1 \mathrm{H}), 3.66(\mathrm{~m}, 3 \mathrm{H}), 3.96(\mathrm{~m}, 3 \mathrm{H})$, 5.06 (br d, 1H, NH), 5.17 (s, 2H), 7.21-7.39 (m, 16H), 7.63-7.67 (m, 4H) ppm. ${ }^{13} \mathrm{C}-\mathrm{NMR}(100$ $\left.\mathrm{MHz}, \mathrm{CDCl}_{3}\right): \delta=19.4(\mathrm{C}), 23.1\left(\mathrm{CH}_{2}\right), 27.1\left(\mathrm{CH}_{3}\right), 29.1\left(\mathrm{CH}_{2}\right), 39.0\left(\mathrm{CH}_{2}\right), 40.8\left(\mathrm{CH}_{2}\right), 60.2$ $(\mathrm{CH}), 63.7\left(\mathrm{CH}_{2}\right), 67.1\left(\mathrm{CH}_{2}\right), 72.0(\mathrm{CH}), 72.3(\mathrm{CH}), 80.2(\mathrm{C}), 127.0(\mathrm{CH}), 127.9(\mathrm{CH}), 128.3$ $(\mathrm{CH}), 128.4(\mathrm{CH}), 128.8(\mathrm{CH}), 129.4(\mathrm{CH}), 129.8(\mathrm{CH}), 130.4(\mathrm{CH}), 134.0(\mathrm{C}), 134.1(\mathrm{C}), 135.8$ $(\mathrm{CH}), 136.6(\mathrm{C}), 156.8(\mathrm{C}) \mathrm{ppm}$. MS (FAB $\left.{ }^{+}, \mathrm{NBA}\right) \mathrm{m} / e: 674\left([\mathrm{M}+23]^{+}, 100 \%\right), 652\left([\mathrm{M}+1]^{+}\right.$, 47\%). HRMS (FAB $\left.{ }^{+}\right) \mathrm{C}_{39} \mathrm{H}_{46} \mathrm{NO}_{4} \mathrm{SSi}(\mathrm{M}+1)$ : 652.2917 calcd., 652.2920 found.

\section{(2S,3S)-3-(Benzyloxycarbonyl-(2-propynyl)amino)-6-(tert-butyldiphenylsilyloxy)-1-}

phenylsulfonyl-2-hexanol (11b). To a solution of the hydroxysulfide $10 \mathrm{~b}(1.3 \mathrm{~g}, 2.0 \mathrm{mmol})$ in anhydrous dichloromethane $(40 \mathrm{ml})$, a solution of $m$-CPBA $(0.90 \mathrm{~g}, 5.0 \mathrm{mmol})$ in dry dichloromethane $(40 \mathrm{ml})$ was added dropwise. The reaction mixture was stirred at room temperature until total disappearance of the starting sulfide ( $2 \mathrm{~h}$, TLC monitoring), and cooled to $0^{\circ} \mathrm{C}$. After addition of a $10 \%$ aqueous solution of sodium sulfite $(30 \mathrm{ml})$, stirring was maintained at the same temperature for $15 \mathrm{~min}$. The organic phase was separated and washed with a 
saturated solution of sodium bicarbonate $(3 \times 15 \mathrm{ml})$ and with brine $(30 \mathrm{ml})$. The organic phase was dried over anhydrous $\mathrm{MgSO}_{4}$ and the solvent was removed under vacuum. Column chromatography of the crude product $(2.5 \% \mathrm{v} / \mathrm{v}$ triethylamine-pretreated silica gel, hexane-ethyl acetate mixtures as eluents) afforded $1.30 \mathrm{~g}$ (95\% yield) of $(2 S, 3 S)$-3-(benzyloxycarbonyl-(2propynyl)amino)-6-(tert-butyldiphenylsilyloxy)-1-phenylsulfonyl-2-hexanol $\mathbf{1 1 b}$ as a dense, colorless oil. $[\alpha]_{\mathrm{D}}^{23}=-2.5\left(c=1.0, \mathrm{CHCl}_{3}\right)$. IR $(\mathrm{NaCl}$ film $): v_{\max }=2933,2858,1700,1447$, 1428, 1306, 1250, 1144, 1111, 743, 702, $687 \mathrm{~cm}^{-1} .{ }^{1} \mathrm{H}-\mathrm{NMR}\left(400 \mathrm{MHz}, \mathrm{CDCl}_{3}\right): \delta=1.02(\mathrm{~s}$, $9 \mathrm{H}), 1.46-1.74(\mathrm{~m}, 4 \mathrm{H}), 2.00(\mathrm{~s}, 1 \mathrm{H}), 3.22-3.37(\mathrm{~m}, 2 \mathrm{H}), 3.62(\mathrm{~m}, 2 \mathrm{H}), 3.79-4.12(\mathrm{~m}, 3 \mathrm{H}), 4.30$ $(\mathrm{m}, 1 \mathrm{H}), 5.13(\mathrm{~s}, 2 \mathrm{H}), 7.34-7.38(\mathrm{~m}, 10 \mathrm{H}), 7.55-7.68(\mathrm{~m}, 8 \mathrm{H}), 7.89-7.93(\mathrm{~m}, 2 \mathrm{H}) \mathrm{ppm} .{ }^{13} \mathrm{C}-\mathrm{NMR}$ $\left(100 \mathrm{MHz}, \mathrm{CDCl}_{3}\right): \delta=19.4(\mathrm{C}), 26.2\left(\mathrm{CH}_{2}\right), 27.1\left(\mathrm{CH}_{3}\right), 28.8\left(\mathrm{CH}_{2}\right), 34.2\left(\mathrm{CH}_{2}\right), 60.0\left(\mathrm{CH}_{2}\right)$, $61.1(\mathrm{CH}), 63.7\left(\mathrm{CH}_{2}\right), 68.0\left(\mathrm{CH}_{2}\right), 68.5(\mathrm{CH}), 72.1(\mathrm{CH}), 80.0(\mathrm{C}), 127.8(\mathrm{CH}), 128.2(\mathrm{CH})$, $128.3(\mathrm{CH}), 128.8(\mathrm{CH}), 128.9(\mathrm{CH}), 129.7(\mathrm{CH}), 129.9(\mathrm{CH}), 134.2(\mathrm{C}), 135.7(\mathrm{CH}), 135.8$ (CH), $136.3(\mathrm{C}), 139.4(\mathrm{C}), 156.4(\mathrm{C}) \mathrm{ppm}$. MS (FAB $\left.{ }^{+}, \mathrm{NBA}\right) \mathrm{m} / e: 706\left([\mathrm{M}+23]^{+}, 100 \%\right), 684$ $\left([\mathrm{M}+1]^{+}, 30 \%\right), 640\left([\mathrm{M}-43]^{+}, 65 \%\right)$. HRMS $\left(\mathrm{FAB}^{+}\right) \mathrm{C}_{39} \mathrm{H}_{46} \mathrm{NO}_{6} \mathrm{SSi}(\mathrm{M}+1)$ : 684.2815 calcd., 684.2837 found.

\section{(3S,E)-3-(Benzyloxycarbonyl-(2-propynyl)amino)-6-(tert-butyldiphenylsilyloxy)-1-}

phenylsulfonyl-1-hexene (2b). A solution of the hydroxysulfone $\mathbf{1 1 b}(1.30 \mathrm{~g}, 1.90 \mathrm{mmol})$, the water-soluble carbodiimide morpho-CDI (1.60 g, $3.80 \mathrm{mmol})$ and anhydrous copper(II) chloride $(28 \mathrm{mg}, 0.19 \mathrm{mmol})$ in dry acetonitrile $(50 \mathrm{ml})$ was heated to $70^{\circ} \mathrm{C}$ and stirred during $16 \mathrm{~h}$. After cooling to room temperature, the reaction mixture was filtered through a short pad or Celite ${ }^{\circledR}$ and silica gel, that was thoroughly washed with $\mathrm{CH}_{2} \mathrm{Cl}_{2}$. Removal of solvents under vacuum gave $1.30 \mathrm{~g}$ (quantitative yield) of pure (3S,E)-3-(benzyloxycarbonyl-(2-propynyl)amino)-6(tert-butyldiphenylsilyloxy)-1-phenylsulfonyl-1-hexene $\mathbf{2 b}$ as a dense, colorless oil. $[\alpha]_{\mathrm{D}}{ }^{23}=-8.0$ $\left(c=1.0, \mathrm{CHCl}_{3}\right)$. IR ( $\mathrm{NaCl}$ film): $v_{\max }=3288,2931,2858,1702,1447,1428,1322,1148,1111$, 1086, 702, $689 \mathrm{~cm}^{-1} .{ }^{1} \mathrm{H}-\mathrm{NMR}\left(400 \mathrm{MHz}, \mathrm{CDCl}_{3}\right): \delta=1.03(\mathrm{~s}, 9 \mathrm{H}), 1.57(\mathrm{~m}, 2 \mathrm{H}), 1.82(\mathrm{~m}, 2 \mathrm{H})$, 2.12 (s, 1H), 3.63 (br m, 2H), 3.94 (br m, 2H), 4.60 (br m, 1H), 5.13 (br m, 2H), 6.38 (m, 1H), $6.98\left(\mathrm{dd}, J_{1}=15.0 \mathrm{~Hz}, J_{2}=5.5 \mathrm{~Hz}, 1 \mathrm{H}\right), 7.34-7.38(\mathrm{~m}, 10 \mathrm{H}), 7.53-7.62(\mathrm{~m}, 8 \mathrm{H}), 7.89-7.93(\mathrm{~m}$, 2H) ppm. ${ }^{13} \mathrm{C}-\mathrm{NMR}\left(100 \mathrm{MHz}, \mathrm{CDCl}_{3}\right): \delta=19.2(\mathrm{C}), 27.0\left(\mathrm{CH}_{3}\right), 29.0\left(\mathrm{CH}_{2}\right), 29.7\left(\mathrm{CH}_{2}\right), 33.5$ $\left(\mathrm{CH}_{2}\right), 56.2(\mathrm{CH}), 62.8\left(\mathrm{CH}_{2}\right), 67.7\left(\mathrm{CH}_{2}\right), 72.2(\mathrm{CH}), 80.0(\mathrm{C}), 127.6(\mathrm{CH}), 127.7(\mathrm{CH}), 128.1$ $(\mathrm{CH}), 128.3(\mathrm{CH}), 128.5(\mathrm{CH}), 129.2(\mathrm{CH}), 129.6(\mathrm{CH}), 131.8(\mathrm{CH}), 133.4(\mathrm{CH}), 133.7(\mathrm{C})$, $135.1(\mathrm{C}), 135.4(\mathrm{CH}), 139.9(\mathrm{C}), 144.2(\mathrm{CH}), 156.6(\mathrm{C}) \mathrm{ppm}$. MS (FAB ${ }^{+}$, glycerol) m/e: 666 $\left([\mathrm{M}+1]^{+}, 20 \%\right), 622\left([\mathrm{M}-43]^{+}, 30 \%\right), 608\left([\mathrm{M}-57]^{+}, 50 \%\right), 588\left([\mathrm{M}-77]^{+}, 100 \%\right)$. HRMS (ESI) $\mathrm{C}_{39} \mathrm{H}_{43} \mathrm{NNaO}_{5} \mathrm{SSi}(\mathrm{M}+\mathrm{Na})$ : 688.2523 calcd., 688.2525 found.

(2R,3R)-2,3-Epoxy-3-methyl-1-propanol (12c). Prepared from freshly distilled trans-crotyl alcohol (6.3 ml, $75.0 \mathrm{mmol})$ by catalytic asymmetric epoxidation (D-(-)-DIPT), as described in ref. 30. Yield: 53\% (3.5 g). ee: 93\% ( ${ }^{1} \mathrm{H}-\mathrm{NMR}$, Mosher's ester). ${ }^{31}{ }^{1} \mathrm{H}-\mathrm{NMR}\left(400 \mathrm{MHz}, \mathrm{CDCl}_{3}\right)$ : $\delta=1.34(\mathrm{~d}, J=5.2 \mathrm{~Hz}, 3 \mathrm{H}), 2.35($ br s, $1 \mathrm{H}, \mathrm{OH}), 2.90(\mathrm{~m}, 1 \mathrm{H}), 3.03(\mathrm{~m}, 1 \mathrm{H}), 3.60\left(\mathrm{dd}, J_{1}=12.4\right.$ $\left.\mathrm{Hz}, J_{2}=3.6 \mathrm{~Hz}, 1 \mathrm{H}\right), 3.90\left(\mathrm{dd}, J_{1}=12.4 \mathrm{~Hz}, J_{2}=1.2 \mathrm{~Hz}, 1 \mathrm{H}\right) \mathrm{ppm} .{ }^{13} \mathrm{C}-\mathrm{NMR}\left(100 \mathrm{MHz}, \mathrm{CDCl}_{3}\right)$ : $\delta=17.1\left(\mathrm{CH}_{3}\right), 52.0(\mathrm{CH}), 59.5(\mathrm{CH}), 61.6\left(\mathrm{CH}_{2}\right) \mathrm{ppm}$. 
(2R,3R)-2,3-Epoxy-3-phenyl-1-propanol (12d). Prepared from freshly distilled trans-cinnamyl alcohol $(2.7 \mathrm{~g}, 20.0 \mathrm{mmol})$ by catalytic asymmetric epoxidation (D-(-)-DIPT), as described in ref. 10b. Yield: $84 \%(2.5 \mathrm{~g})$. ee: $>98 \%$ (polarimetry). Mp $54-55^{\circ} \mathrm{C}\left(\right.$ (lit: $\left.^{10 \mathrm{~b}} 51.5-53^{\circ} \mathrm{C}\right) \cdot[\alpha]_{\mathrm{D}}{ }^{23}=$ $+50.4\left(c=2.4, \mathrm{CHCl}_{3}\right)\left[\mathrm{lit}(98 \%\right.$ ee $\left.):^{10 \mathrm{~b}}[\alpha]_{\mathrm{D}}^{25}=+49.6\left(c=2.4, \mathrm{CHCl}_{3}\right)\right]$.

(2S,3S)-3-Azido-1,2-butanediol (5c). ${ }^{32}$ A solution of titanium tetra(isopropoxide) (11.2 ml, 37.5 $\mathrm{mmol})$ and of azidotrimethylsilane $(10.0 \mathrm{ml}, 75.0 \mathrm{mmol})$ in anhydrous toluene $(125 \mathrm{ml})$ was stirred at $90^{\circ} \mathrm{C}$ during $5 \mathrm{~h}$ under argon. After cooling to $75^{\circ} \mathrm{C}$, a solution of epoxyalcohol $12 \mathrm{c}(2.2$ $\mathrm{g}, 25.0 \mathrm{mmol})$ in dry toluene $(50 \mathrm{ml})$ was added dropwise. Stirring was maintained at the same temperature for $1 \mathrm{~h}$ (TLC monitoring). After cooling to room temperature, a $10 \%$ aqueous solution of $\mathrm{NaOH}$ in brine $(75 \mathrm{ml})$ was added, and the resulting mixture was stirred overnight. After filtration through Celite ${ }^{\circledR}$, the organic phase was dried over anhydrous magnesium sulfate. Evaporation of the solvent under vacuum afforded $2.4 \mathrm{~g}$ (75\% yield) of (2S,3S)-3-azido-1,2butanediol $\mathbf{5 c}$ as a colorless oil, that was used for the next step without further purification. $[\alpha]_{\mathrm{D}}{ }^{23}=+61.8\left(c=1.05, \mathrm{CHCl}_{3}\right) . \mathrm{IR}(\mathrm{NaCl}$ film $): v_{\max }=3363,2977,2937,2117,1457,1382$, 1258, 1115, $1042 \mathrm{~cm}^{-1} .{ }^{1} \mathrm{H}-\mathrm{NMR}\left(400 \mathrm{MHz}, \mathrm{CDCl}_{3}\right): \delta=1.30(\mathrm{~d}, J=6.4 \mathrm{~Hz}, 3 \mathrm{H}), 3.40(\mathrm{br} \mathrm{s}, 2 \mathrm{H}$, 2OH), 3.55-3.71 (m, 4H) ppm. ${ }^{13} \mathrm{C}-\mathrm{NMR}\left(100 \mathrm{MHz}, \mathrm{CDCl}_{3}\right): \delta=14.8\left(\mathrm{CH}_{3}\right), 58.8(\mathrm{CH}), 63.1$ $\left(\mathrm{CH}_{2}\right), 74.4(\mathrm{CH}) \mathrm{ppm}$. MS (CI, NH $\left.\mathrm{N}_{3}\right)$ m/e: $149\left([\mathrm{M}+18]^{+}, 100 \%\right), 132\left([\mathrm{M}+1]^{+}, 15 \%\right), 106(\mathrm{M}-$ $25,48 \%)$.

(2S,3S)-3-Azido-3-phenyl-1,2-propanediol (5d). ${ }^{33}$ A mixture of epoxyalcohol 12d $(0.75 \mathrm{~g}, 5.0$ $\mathrm{mmol})$, lithium perchlorate $(13.0 \mathrm{~g}, 125 \mathrm{mmol})$ and sodium azide $(1.6 \mathrm{~g}, 25 \mathrm{mmol})$ in dry acetonitrile $(25 \mathrm{ml})$ was stirred at $65{ }^{\circ} \mathrm{C}$ for $5 \mathrm{~h}$ (TLC monitoring). After cooling to room temperature, the reaction mixture was poured over water $(310 \mathrm{ml})$. The aqueous phase was extracted with diethyl ether $(3 \times 50 \mathrm{ml})$, and the organic extracts were dried over anhydrous magnesium sulfate. Evaporation of the solvent under vacuum afforded $0.91 \mathrm{~g}$ (94\% yield) of highly pure (2S,3S)-3-azido-3-phenyl-1,2-propanediol 5d. ${ }^{1} \mathrm{H}-\mathrm{NMR}\left(200 \mathrm{MHz}, \mathrm{CDCl}_{3}\right): \delta=2.23$ (br s, 2H, 2OH), $3.73(\mathrm{~m}, 2 \mathrm{H}), 3.81(\mathrm{~m}, 1 \mathrm{H}), 4.64(\mathrm{~d}, J=7.0 \mathrm{~Hz}, 1 \mathrm{H}), 7.37-7.41(\mathrm{~m}, 5 \mathrm{H}) \mathrm{ppm}$.

(2S,3S)-3-(Benzyloxycarbonylamino)-1,2-butanediol (6c). ${ }^{32}$ A solution of azidodiol 5c $(3.40$ $\mathrm{g}, 26.0 \mathrm{mmol})$ in anhydrous $\mathrm{MeOH}(100 \mathrm{ml})$ was stirred at room temperature under hydrogen (balloon) in the presence of $10 \% \mathrm{Pd} / \mathrm{C}(0.34 \mathrm{~g})$ during $2.5 \mathrm{~h}$ (TLC monitoring). After filtration through Celite ${ }^{\mathbb{R}}$ and removal of the solvent (2S,3S)-3-amino-1,2-butanediol (2.80 g, quantitative yield) was obtained. ${ }^{1} \mathrm{H}-\mathrm{NMR}\left(200 \mathrm{MHz}, \mathrm{CD}_{3} \mathrm{OD}\right): \delta=1.13\left(\mathrm{dd}, J_{1}=6.6 \mathrm{~Hz}, J_{2}=3.0 \mathrm{~Hz}, 3 \mathrm{H}\right)$, $2.96(\mathrm{~m}, 1 \mathrm{H}), 3.50(\mathrm{~m}, 1 \mathrm{H}), 3.61(\mathrm{~m}, 2 \mathrm{H}) \mathrm{ppm}$.

A solution of this crude aminodiol $(2.8 \mathrm{~g}, 26.0 \mathrm{mmol})$ in anhydrous tetrahydrofuran $(50 \mathrm{ml})$ was treated with triethylamine $(4.3 \mathrm{ml}, 31.2 \mathrm{mmol}), 4-D M A P(312 \mathrm{mg}, 2.6 \mathrm{mmol})$ and benzyl chloroformate ( $4.4 \mathrm{ml}, 31.2 \mathrm{mmol})$ for $2 \mathrm{~h}$ at room temperature (TLC monitoring). The resulting solution was washed with brine $(80 \mathrm{ml})$, and the aqueous phase was extracted with diethyl ether ( $3 \times 40 \mathrm{ml}$ ). The combined organic phases were dried over anhydrous $\mathrm{MgSO}_{4}$ and the solvents were removed under vacuum. Column chromatography of the crude product $(2.5 \% \mathrm{v} / \mathrm{v}$ triethylamine-pretreated silica gel, hexane-ethyl acetate mixtures as eluents) afforded $3.4 \mathrm{~g} \mathrm{(55 \%}$ yield from 12c) of the title compound, as a colorless oil. ${ }^{1} \mathrm{H}-\mathrm{NMR}\left(200 \mathrm{MHz}, \mathrm{CDCl}_{3}\right): \delta=1.21$ 
$(\mathrm{d}, J=6.6 \mathrm{~Hz}, 3 \mathrm{H}), 3.42$ (br m, 1H), $3.62(\mathrm{~m}, 2 \mathrm{H}), 3.74(\mathrm{~m}, 1 \mathrm{H}), 5.09$ (br s, $2 \mathrm{H}+\mathrm{NH}), 7.34-7.37$ $(\mathrm{m}, 5 \mathrm{H}) \mathrm{ppm}$.

(2S,3S)-3-(Benzyloxycarbonylamino)-3-phenyl-1,2-propanediol (6d). A solution of azidodiol 5d (4.60 g, $23.5 \mathrm{mmol})$ in dry ethyl acetate $(190 \mathrm{ml})$ was stirred at room temperature under hydrogen (balloon) in the presence of $10 \% \mathrm{Pd} / \mathrm{C}(0.46 \mathrm{~g})$ during $2 \mathrm{~h}$ (TLC monitoring). After filtration through Celite ${ }^{\circledR}$ and removal of the solvent (2S,3S)-3-amino-3-phenyl-1,2-propanediol (4.0 g, quantitative yield) was obtained. ${ }^{1} \mathrm{H}-\mathrm{NMR}\left(200 \mathrm{MHz}, \mathrm{CD}_{3} \mathrm{OD}\right): \delta=3.04$ (br, $3 \mathrm{H}, 2 \mathrm{OH}+$ $\mathrm{NH}), 3.55(\mathrm{~m}, 2 \mathrm{H}), 3.78(\mathrm{~m}, 1 \mathrm{H}), 4.10(\mathrm{~m}, 1 \mathrm{H}), 7.31-7.33(\mathrm{~m}, 5 \mathrm{H}) \mathrm{ppm}$.

A solution of this crude aminodiol $(4.00 \mathrm{~g}, 23.5 \mathrm{mmol})$ in anhydrous tetrahydrofuran $(50 \mathrm{ml})$ was treated with triethylamine $(4.0 \mathrm{ml}, 28.2 \mathrm{mmol}), 4-D M A P(280 \mathrm{mg}, 2.4 \mathrm{mmol})$ and benzyl chloroformate $(4.1 \mathrm{ml}, 28.2 \mathrm{mmol})$ for $2 \mathrm{~h}$ at room temperature (TLC monitoring). The resulting solution was washed with brine $(80 \mathrm{ml})$, and the aqueous phase was extracted with diethyl ether $(3 \times 40 \mathrm{ml})$. The combined organic phases were dried over anhydrous $\mathrm{MgSO}_{4}$ and the solvents were removed under vacuum. Column chromatography of the crude product $(2.5 \% \mathrm{v} / \mathrm{v}$ triethylamine-pretreated silica gel, hexane-ethyl acetate mixtures as eluents) afforded $3.70 \mathrm{~g}$ (53\% yield from 12d) of (2S,3S)-3-(benzyloxycarbonylamino)-3-phenyl-1,2-propanediol $6 \mathbf{d}$ as a colorless solid. Mp $103-104^{\circ} \mathrm{C} .[\alpha]_{\mathrm{D}}^{23}=+33.1\left(c=1.0, \mathrm{CHCl}_{3}\right)$. IR $\left(\mathrm{NaCl}\right.$ film): $v_{\max }=3327$, 3034, 2945, 1688, 1539, 1454, 1285, 1259, 1106, 1052, 1014, 753, $697 \mathrm{~cm}^{-1}$. ${ }^{1} \mathrm{H}-\mathrm{NMR}(400$ $\mathrm{MHz}_{\mathrm{CDCl}}$ ): $\delta=2.83$ (br, 2H, 2OH), 3.52 (br d, 1H), 3.61 (br d, 1H), $3.85(\mathrm{~m}, 1 \mathrm{H}), 4.79(\mathrm{~m}$, 1H), $5.05(\mathrm{~m}, 2 \mathrm{H}), 5.78$ (br d, 1H, NH), 7.27-7.34 (m, 10H) ppm. ${ }^{13} \mathrm{C}-\mathrm{NMR}\left(100 \mathrm{MHz}, \mathrm{CDCl}_{3}\right)$ : $\delta=57.4(\mathrm{CH}), 63.2\left(\mathrm{CH}_{2}\right), 67.2\left(\mathrm{CH}_{2}\right), 73.7(\mathrm{CH}), 127.3(\mathrm{CH}), 128.0(\mathrm{CH}), 128.2(\mathrm{CH}), 128.5$ $(\mathrm{CH}), 128.8(\mathrm{CH}), 136.1(\mathrm{C}), 138.5(\mathrm{C}), 156.6(\mathrm{C}) \mathrm{ppm}$. MS (CI, NH$)$ m/e: $319\left([\mathrm{M}+18]^{+}, 45 \%\right)$, $302\left([\mathrm{M}+1]^{+}, 100 \%\right), 258\left([\mathrm{M}-43]^{+}, 20 \%\right)$. Anal. $\left(\mathrm{C}_{17} \mathrm{H}_{19} \mathrm{NO}_{4}\right)$, (calcd., found): \%C (67.76, $67.22), \% \mathrm{H}(6.36,6.46), \% \mathrm{~N}(4.65,4.66)$.

$(\boldsymbol{S})-2-[(\boldsymbol{S})$-1-(Benzyloxycarbonylamino)ethyl $]$ oxirane $(\mathbf{4 c}) .^{34}$ To a solution of diol $\mathbf{6 c}(3.30 \mathrm{~g}$, $13.8 \mathrm{mmol})$ and of triphenylphosphine $(3.9 \mathrm{~g}, 15 \mathrm{mmol})$ in anhydrous chloroform $(55 \mathrm{ml})$, a solution of diisopropylazodicarboxylate $(2.9 \mathrm{ml}, 15 \mathrm{mmol})$ in anhydrous chloroform $(15 \mathrm{ml})$ was added dropwise. The resulting mixture was heated to reflux until total disappearance of the starting diol (18 h, TLC monitoring). The solvent was removed at reduced pressure and the residue was purified by column chromatography (silica gel, hexane-ethyl acetate mixtures as eluents) to afford $2.20 \mathrm{~g}$ (72\% yield) of $(S)$-2-[(S)-1-(benzyloxycarbonylamino)ethyl]oxirane $4 \mathbf{c}$. ${ }^{1} \mathrm{H}-\mathrm{NMR}\left(400 \mathrm{MHz}, \mathrm{CDCl}_{3}\right): \delta=1.17(\mathrm{~d}, J=6.8 \mathrm{~Hz}, 3 \mathrm{H}), 2.74(\mathrm{br} \mathrm{d}, 2 \mathrm{H}), 2.93(\mathrm{~m}, 1 \mathrm{H}), 3.72$ (br $\mathrm{m}, 1 \mathrm{H}), 4.92(\mathrm{br} \mathrm{s}, 1 \mathrm{H}, \mathrm{NH}), 5.09(\mathrm{~s}, 2 \mathrm{H}), 7.30-7.38(\mathrm{~m}, 5 \mathrm{H}) \mathrm{ppm} .{ }^{13} \mathrm{C}-\mathrm{NMR}\left(100 \mathrm{MHz}, \mathrm{CDCl}_{3}\right)$ : $\delta=16.3\left(\mathrm{CH}_{3}\right), 46.0\left(\mathrm{CH}_{2}\right), 47.8(\mathrm{CH}), 54.3(\mathrm{CH}), 66.7\left(\mathrm{CH}_{2}\right), 128.0(\mathrm{CH}), 128.1(\mathrm{CH}), 128.5$ (CH), $136.3(\mathrm{C}), 155.7$ (C) ppm.

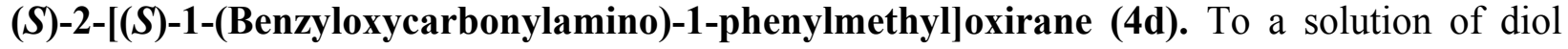
6d (3.70 g, $12.3 \mathrm{mmol})$ and of triphenylphosphine (3.50 g, $13.2 \mathrm{mmol})$ in anhydrous chloroform $(50 \mathrm{ml})$, a solution of diisopropylazodicarboxylate $(2.6 \mathrm{ml}, 13.2 \mathrm{mmol})$ in anhydrous chloroform $(12 \mathrm{ml})$ was added dropwise. The resulting mixture was heated to reflux until total disappearance of the starting diol ( $2 \mathrm{~h}$, TLC monitoring). The solvent was removed at reduced pressure and the 
residue was purified by column chromatography (silica gel, hexane-ethyl acetate mixtures as eluents) to afford $3.25 \mathrm{~g}$ (93\% yield) of $(S)$-2-[(S)-1-(benzyloxycarbonylamino)-1phenylmethyl]oxirane $4 \mathbf{d}$ as a colorless solid. $\mathrm{Mp} 72-74^{\circ} \mathrm{C} .[\alpha]_{\mathrm{D}}{ }^{23}=+13.4\left(c=1.0, \mathrm{CHCl}_{3}\right)$. IR $\left(\mathrm{NaCl}\right.$ film): $v_{\max }=3324,3033,1702,1528,1454,1400,1234,1144,1028,751,699 \mathrm{~cm}^{-1} .{ }^{1} \mathrm{H}-$ NMR (400 MHz, $\left.\mathrm{CDCl}_{3}\right): \delta=2.51($ br m, 1H), 2.75 (br m, 1H), 3.27 (br m, 1H), 4.81 (br m, 1H), $5.09(\mathrm{~m}, 2 \mathrm{H}), 5.33(\mathrm{br} \mathrm{s}, 1 \mathrm{H}, \mathrm{NH}), 7.27-7.36(\mathrm{~m}, 10 \mathrm{H}) \mathrm{ppm} .{ }^{13} \mathrm{C}-\mathrm{NMR}\left(100 \mathrm{MHz}, \mathrm{CDCl}_{3}\right): \delta=$ $45.7\left(\mathrm{CH}_{2}\right), 53.6(\mathrm{CH}), 55.8(\mathrm{CH}), 67.0\left(\mathrm{CH}_{2}\right), 127.1(\mathrm{CH}), 128.1(\mathrm{CH}), 128.2(\mathrm{CH}), 128.5(\mathrm{CH})$, 128.6 (CH), $128.7(\mathrm{CH}), 136.1(\mathrm{C}), 137.8(\mathrm{C}), 155.7(\mathrm{C}) \mathrm{ppm}$. MS (CI, NH$\left.)_{3}\right) \mathrm{m} / \mathrm{e}: 301\left([\mathrm{M}+18]^{+}\right.$, $37 \%), 284\left([\mathrm{M}+1]^{+}, 100 \%\right), 240\left([\mathrm{M}-43]^{+}, 43 \%\right)$. Anal. $\left(\mathrm{C}_{17} \mathrm{H}_{17} \mathrm{NO}_{3}\right)$, (calcd., found): $\% \mathrm{C}$ $(72.07,71.67), \% \mathrm{H}(6.05,6.19), \% \mathrm{~N}(4.94,5.32)$.

(S)-2-[(S)-1-(Benzyloxycarbonyl-(2-propynyl)amino)ethyl]oxirane $\quad(9 \mathrm{c}) . \quad$ To $\quad$ a stirred suspension of sodium hydride $(0.72 \mathrm{~g}, 60 \%$ in paraffines, $18.0 \mathrm{mmol})$ in anhydrous tetrahydrofuran $(25 \mathrm{ml})$, a solution of oxirane $4 \mathbf{c}(0.80 \mathrm{~g}, 3.6 \mathrm{mmol})$ in anhydrous tetrahydrofuran $(25 \mathrm{ml})$ was added dropwise, and stirring was maintained at room temperature for $15 \mathrm{~min}$. At this point, propargyl bromide $(3.9 \mathrm{ml}, 36 \mathrm{mmol})$ and HMPA $(6.3 \mathrm{ml}, 26 \mathrm{mmol})$ were added sequentially with the aid of a syringe. The resulting mixture was heated to reflux for $3 \mathrm{~h}$. After cooling to room temperature, a saturated brine solution $(15 \mathrm{ml})$ was added and stirring maintained for $5 \mathrm{~min}$. The reaction mixture was extracted with diethyl ether $(3 \times 10 \mathrm{ml})$; the organic extracts were dried over magnesium sulfate and the solvents removed at reduced pressure. Column chromatography of the crude product (silica gel, hexane-ethyl acetate mixtures as eluents) afforded $0.50 \mathrm{~g} \quad(54 \%$ yield $)$ of $(S)-2-[(S)-1$-(benzyloxycarbonyl-(2propynyl)amino)ethyl]oxirane $9 \mathrm{c}$ as a colorless oil. $[\alpha]_{\mathrm{D}}{ }^{23}=-12.6\left(c=1.0, \mathrm{CHCl}_{3}\right)$. IR $(\mathrm{NaCl}$ film): $v_{\max }=3287,2984,1701,1451,1409,1368,1321,1257,1200,1114,738,698 \mathrm{~cm}^{-1} .{ }^{1} \mathrm{H}-$ NMR (400 MHz, $\left.\mathrm{CDCl}_{3}\right): \delta=1.25(\mathrm{~d}, J=6.8 \mathrm{~Hz}, 3 \mathrm{H}), 2.21(\mathrm{t}, J=2.0 \mathrm{~Hz}, 1 \mathrm{H}), 2.60(\mathrm{br} \mathrm{m}, 1 \mathrm{H})$, 2.72 (br m, 1H), 3.18 (br m, 1H), 4.11 (br m, 3H), 5.19 (s, 2H), 7.34-7.38 (m, 5H) ppm. ${ }^{13} \mathrm{C}-$ NMR (100 MHz, $\left.\mathrm{CDCl}_{3}\right): \delta=13.0\left(\mathrm{CH}_{3}\right), 33.3\left(\mathrm{CH}_{2}\right), 45.1\left(\mathrm{CH}_{2}\right), 52.1(\mathrm{CH}), 53.5(\mathrm{CH}), 67.6$ $\left(\mathrm{CH}_{2}\right), 71.1(\mathrm{CH}), 80.6(\mathrm{C}), 127.6(\mathrm{CH}), 128.1(\mathrm{CH}), 128.5(\mathrm{CH}), 136.3(\mathrm{C}), 155.4(\mathrm{C}) \mathrm{ppm} . \mathrm{MS}$ $\left(\mathrm{CI}, \mathrm{NH}_{3}\right) \mathrm{m} / \mathrm{e}: 277\left([\mathrm{M}+18]^{+}, 29 \%\right), 260\left([\mathrm{M}+1]^{+}, 100 \%\right), 216\left([\mathrm{M}-43]^{+}, 18 \%\right)$. HRMS (CI, $\left.\mathrm{NH}_{3}\right) \mathrm{C}_{15} \mathrm{H}_{18} \mathrm{NO}_{3}(\mathrm{M}+1)$ : 260.1286 calcd., 260.1279 found.

(S)-2-[(S)-1-(Benzyloxycarbonyl-(2-propynyl)amino)-1-phenylmethyl]oxirane (9d). To a stirred suspension of sodium hydride $(0.70 \mathrm{~g}, 60 \%$ in paraffines, $17.7 \mathrm{mmol})$ in anhydrous tetrahydrofuran $(20 \mathrm{ml})$, a solution of oxirane $4 \mathbf{d}(1.00 \mathrm{~g}, 3.5 \mathrm{mmol})$ in anhydrous tetrahydrofuran $(20 \mathrm{ml})$ was added dropwise, and stirring was maintained at room temperature for $15 \mathrm{~min}$. At this point, propargyl bromide (3.8 $\mathrm{ml}, 35 \mathrm{mmol})$ and HMPA (12.2 ml, $70 \mathrm{mmol})$ were added sequentially with the aid of a syringe. The resulting mixture was heated to reflux for $1 \mathrm{~h}$. After cooling to room temperature, a saturated brine solution $(20 \mathrm{ml})$ was added and stirring maintained for $5 \mathrm{~min}$. The reaction mixture was extracted with diethyl ether $(3 \times 15 \mathrm{ml})$; the organic extracts were dried over magnesium sulfate and the solvents removed at reduced pressure. Column chromatography of the crude product (silica gel, hexane-ethyl acetate mixtures as eluents) afforded $810 \mathrm{mg} \quad(72 \%$ yield) of $(S)-2-[(S)-1$-(benzyloxycarbonyl-(2- 
propynyl)amino)-1-phenylmethyl]oxirane 9d as a colorless oil. $[\alpha]_{\mathrm{D}}^{23}=+1.1\left(c=1.0, \mathrm{CHCl}_{3}\right)$. IR (NaCl film): $v_{\max }=3290,3033,1702,1497,1452,1406,1366,1249,1124,739,698 \mathrm{~cm}^{-1}$. ${ }^{1} \mathrm{H}-\mathrm{NMR}\left(400 \mathrm{MHz}, \mathrm{CDCl}_{3}\right): \delta=2.22(\mathrm{t}, J=2.4 \mathrm{~Hz}, 1 \mathrm{H}), 2.76$ (br m, 1H), 2.87 (br m, 1H), 3.68 (br m, 1H), 3.84 (br d, 1H), 4.21 (br d, 1H), 4.82 (br m, 1H), 5.19 (s, 2H), 7.27-7.40 (m, 10H) ppm. ${ }^{13} \mathrm{C}-\mathrm{NMR}\left(100 \mathrm{MHz}, \mathrm{CDCl}_{3}\right): \delta=35.0\left(\mathrm{CH}_{2}\right), 46.8\left(\mathrm{CH}_{2}\right), 52.1(\mathrm{CH}), 62.6(\mathrm{CH}), 68.1$ $\left(\mathrm{CH}_{2}\right), 71.9(\mathrm{CH}), 80.5(\mathrm{C}), 127.7(\mathrm{CH}), 128.1(\mathrm{CH}), 128.3(\mathrm{CH}), 128.4(\mathrm{CH}), 128.7(\mathrm{CH}), 128.9$ (CH), 136.1 (C), 137.8 (C), 155.9 (C) ppm. MS (CI, NH $\mathrm{NH}_{3}$ m/e: 339 ([M+18] $\left.]^{+}, 4 \%\right), 322$ $\left([\mathrm{M}+1]^{+}, 100 \%\right), 278\left([\mathrm{M}-43]^{+}, 66 \%\right)$. HRMS (CI, $\left.\mathrm{NH}_{3}\right) \mathrm{C}_{20} \mathrm{H}_{20} \mathrm{NO}_{3}(\mathrm{M}+1): 322.1443$ calcd., 322.1439 found.

(2S,3S)-3-(Benzyloxycarbonyl-(2-propynyl)amino)-1-phenylthio-2-butanol (10c). To a solution of oxirane $9 \mathbf{c}(0.44 \mathrm{~g}, 1.7 \mathrm{mmol})$ in anhydrous methanol $(15 \mathrm{ml})$, triethylamine $(240 \mu \mathrm{l}$, $1.7 \mathrm{mmol})$ and thiophenol $(175 \mu \mathrm{l}, 1.7 \mathrm{mmol})$ were added sequentially. The reaction mixture was heated to reflux until total disappearance of the starting oxirane $(1.5 \mathrm{~h}$, TLC monitoring). The solvent was removed at reduced pressure and the residue was purified by column chromatography $(2.5 \% \mathrm{v} / \mathrm{v}$ triethylamine-pretreated silica gel, hexane-ethyl acetate mixtures as eluents) to give $590 \mathrm{mg}$ (94\% yield) of (2S,3S)-3-(benzyloxycarbonyl-(2-propynyl)amino)-1phenylthio-2-butanol 10c as a colorless oil. $[\alpha]_{\mathrm{D}}^{23}=-3.3\left(c=1.0, \mathrm{CHCl}_{3}\right)$. IR $\left(\mathrm{NaCl}\right.$ film): $v_{\max }=$ 3433, 3293, 2939, 1696, 1583, 1440, 1412, 1323, 1256, 1119, 1023, 739, $693 \mathrm{~cm}^{-1} .{ }^{1} \mathrm{H}-\mathrm{NMR}$ $\left(400 \mathrm{MHz}, \mathrm{CDCl}_{3}\right): \delta=1.33(\mathrm{~d}, J=6.8 \mathrm{~Hz}, 3 \mathrm{H}), 2.11(\mathrm{~s}, 1 \mathrm{H}), 2.80-3.18(\mathrm{~m}, 2 \mathrm{H}), 3.93-4.12(\mathrm{~m}$, $4 \mathrm{H}), 5.16(\mathrm{~s}, 2 \mathrm{H}), 7.20-7.36(\mathrm{~m}, 10 \mathrm{H}) \mathrm{ppm} .{ }^{13} \mathrm{C}-\mathrm{NMR}\left(100 \mathrm{MHz}, \mathrm{CDCl}_{3}\right): \delta=12.2\left(\mathrm{CH}_{3}\right), 35.5$ $\left(\mathrm{CH}_{2}\right), 38.9\left(\mathrm{CH}_{2}\right), 57.1(\mathrm{CH}), 67.6\left(\mathrm{CH}_{2}\right), 71.5(\mathrm{CH}), 72.3(\mathrm{CH}), 80.2(\mathrm{C}), 127.7(\mathrm{CH}), 128.1$ $(\mathrm{CH}), 128.5(\mathrm{CH}), 129.1(\mathrm{CH}), 129.8(\mathrm{CH}), 130.5(\mathrm{CH}), 135.1(\mathrm{C}), 136.3(\mathrm{C}), 155.9(\mathrm{C}) \mathrm{ppm}$. MS (CI, $\left.\mathrm{NH}_{3}\right)$ m/e: 370 ([M+1] $\left.]^{+}, 54 \%\right), 279$ ([M-90] $\left.]^{+}, 100 \%\right), 262$ ([M-107] $\left.]^{+}, 50 \%\right)$. HRMS (CI, $\left.\mathrm{NH}_{3}\right) \mathrm{C}_{21} \mathrm{H}_{24} \mathrm{NO}_{3} \mathrm{~S}(\mathrm{M}+1): 370.1477$ calcd., 370.1474 found.

\section{(2S,3S)-3-(Benzyloxycarbonyl-(2-propynyl)amino)-3-phenyl-1-phenylthio-2-propanol}

(10d). To a solution of oxirane 9d $(0.74 \mathrm{~g}, 2.3 \mathrm{mmol})$ in anhydrous methanol $(23 \mathrm{ml})$, triethylamine $(350 \mu \mathrm{l}, 2.5 \mathrm{mmol})$ and thiophenol $(255 \mu \mathrm{l}, 2.5 \mathrm{mmol})$ were added sequentially. The reaction mixture was heated to reflux until total disappearance of the starting oxirane $(1.5 \mathrm{~h}$, TLC monitoring). The solvent was removed at reduced pressure and the residue was purified by column chromatography $(2.5 \% \mathrm{v} / \mathrm{v}$ triethylamine-pretreated silica gel, hexane-ethyl acetate mixtures as eluents) to afford $890 \mathrm{mg}$ (91\% yield) of (2S,3S)-3-(benzyloxycarbonyl-(2propynyl)amino)-3-phenyl-1-phenylthio-2-propanol 10d as a colorless oil. $[\alpha]_{\mathrm{D}}^{23}=+52.5(\mathrm{c}$ $\left.=1.0, \mathrm{CHCl}_{3}\right)$. IR ( $\mathrm{NaCl}$ film): $v_{\max }=3291,3032,1699,1583,1454,1408,1250,1113,740,697$ $\mathrm{cm}^{-1} .{ }^{1} \mathrm{H}-\mathrm{NMR}\left(400 \mathrm{MHz}, \mathrm{CDCl}_{3}\right): \delta=1.97(\mathrm{~s}, 1 \mathrm{H}), 3.02(\mathrm{br} \mathrm{m}, 1 \mathrm{H}), 3.38(\mathrm{br} \mathrm{d}, 1 \mathrm{H}), 3.74(\mathrm{br} \mathrm{m}$, 1H), $3.97\left(\right.$ br m, 1H), $4.54(\mathrm{~m}, 1 \mathrm{H}), 5.22(\mathrm{~m}, 3 \mathrm{H}), 7.21-7.43(\mathrm{~m}, 15 \mathrm{H}) \mathrm{ppm} .{ }^{13} \mathrm{C}-\mathrm{NMR}(100$ $\left.\mathrm{MHz}, \mathrm{CDCl}_{3}\right): \delta=34.2\left(\mathrm{CH}_{2}\right), 39.7\left(\mathrm{CH}_{2}\right), 63.6(\mathrm{CH}), 67.9\left(\mathrm{CH}_{2}\right), 68.8(\mathrm{CH}), 71.8(\mathrm{CH}), 79.9$ (C), $126.8(\mathrm{CH}), 127.7(\mathrm{CH}), 128.1(\mathrm{CH}), 128.2(\mathrm{CH}), 128.5(\mathrm{CH}), 128.6(\mathrm{CH}), 129.0(\mathrm{CH})$, $129.2(\mathrm{CH}), 131.0(\mathrm{C}), 136.3(\mathrm{CH}), 140.0(\mathrm{C}), 155.9$ (C) ppm. MS (CI, $\left.\mathrm{NH}_{3}\right)$ m/e: $432\left([\mathrm{M}+1]^{+}\right.$, 100\%), 431 ([M] $\left.{ }^{+}, 13 \%\right), 341$ ([M-90] $\left.]^{+}, 12 \%\right)$. HRMS (CI, $\left.\mathrm{NH}_{3}\right) \mathrm{C}_{26} \mathrm{H}_{26} \mathrm{NO}_{3} \mathrm{~S}(\mathrm{M}+1): 432.1633$ calcd., 432.1624 found. 
(2S,3S)-3-(Benzyloxycarbonyl-(2-propynyl)amino)-1-phenylsulfonyl-2-butanol (11c). To a solution of the hydroxysulfide $10 \mathrm{c}(0.52 \mathrm{~g}, 1.4 \mathrm{mmol})$ in anhydrous dichloromethane $(30 \mathrm{ml})$, a solution of $m$-CPBA $(0.60 \mathrm{~g}, 3.5 \mathrm{mmol})$ in dry dichloromethane $(30 \mathrm{ml})$ was added dropwise. The reaction mixture was stirred at room temperature until total disappearance of the starting sulfide ( $2 \mathrm{~h}$, TLC monitoring), and cooled to $0^{\circ} \mathrm{C}$. After addition of a $10 \%$ aqueous solution of sodium sulfite $(20 \mathrm{ml})$, stirring was maintained at the same temperature for $15 \mathrm{~min}$. The organic phase was separated and washed with a saturated solution of sodium bicarbonate $(3 \times 10 \mathrm{ml})$ and with brine $(25 \mathrm{ml})$. The organic phase was dried over anhydrous $\mathrm{MgSO}_{4}$ and the solvent was removed under vacuum. Column chromatography of the crude product $(2.5 \% \mathrm{v} / \mathrm{v}$ triethylaminepretreated silica gel, hexane-ethyl acetate mixtures as eluents) afforded $555 \mathrm{mg}$ (quantitative yield) of (2S,3S)-3-(benzyloxycarbonyl-(2-propynyl)amino)-1-phenylsulfonyl-2-butanol 11c as a dense, colorless oil. $[\alpha]_{\mathrm{D}}{ }^{23}=-2.3\left(c=1.0, \mathrm{CHCl}_{3}\right)$. IR $(\mathrm{NaCl}$ film $): v_{\max }=3469,3289,2935$, 1697, 1448, 1412, 1304, 1245, 1145, 1083, 1023, $748 \mathrm{~cm}^{-1} .{ }^{1} \mathrm{H}-\mathrm{NMR}\left(400 \mathrm{MHz}, \mathrm{CDCl}_{3}\right): \delta=$ $1.32(\mathrm{~d}, J=6.8 \mathrm{~Hz}, 3 \mathrm{H}), 2.12$ (br s, $1 \mathrm{H}), 3.29$ (m, 2H), 3.92-4.08 (m, 3H), 4.34 (br m, 1H), 5.12 (s, 2H), 7.33-7.90 (m, 10H) ppm. ${ }^{13} \mathrm{C}-\mathrm{NMR}\left(100 \mathrm{MHz}, \mathrm{CDCl}_{3}\right): \delta=12.9\left(\mathrm{CH}_{3}\right), 34.3\left(\mathrm{CH}_{2}\right), 56.5$ $(\mathrm{CH}), 59.8\left(\mathrm{CH}_{2}\right), 67.6\left(\mathrm{CH}_{2}\right), 68.5(\mathrm{CH}), 71.7(\mathrm{CH}), 80.0(\mathrm{C}), 127.7(\mathrm{CH}), 128.0(\mathrm{CH}), 128.1$ $(\mathrm{CH}), 128.5(\mathrm{CH}), 129.4(\mathrm{CH}), 134.0(\mathrm{CH}), 136.2(\mathrm{C}), 139.2(\mathrm{C}), 155.6(\mathrm{C}) \mathrm{ppm} . \mathrm{MS}\left(\mathrm{CI}, \mathrm{NH}_{3}\right)$ $m / e: 419\left([\mathrm{M}+18]^{+}, 96 \%\right), 402\left([\mathrm{M}+1]^{+}, 100 \%\right), 358([\mathrm{M}-43]+, 59 \%), 311\left([\mathrm{M}-90]^{+}, 69 \%\right), 250$ $\left([\mathrm{M}-151]^{+}, 32 \%\right)$. HRMS (CI, NH$)_{3} \mathrm{C}_{21} \mathrm{H}_{24} \mathrm{NO}_{5} \mathrm{~S}(\mathrm{M}+1)$ : 402.1375 calcd., 402.1373 found.

\section{(2S,3S)-3-(Benzyloxycarbonyl-(2-propynyl)amino)-3-phenyl-1-phenylsulfonyl-2-propanol}

(11d). To a solution of the hydroxysulfide $10 \mathrm{~d}(0.78 \mathrm{~g}, 1.8 \mathrm{mmol})$ in anhydrous dichloromethane (35 ml), a solution of $m$-CPBA $(0.77 \mathrm{~g}, 4.5 \mathrm{mmol})$ in dry dichloromethane $(35 \mathrm{ml})$ was added dropwise. The reaction mixture was stirred at room temperature until total disappearance of the starting sulfide ( $2 \mathrm{~h}$, TLC monitoring), and cooled to $0^{\circ} \mathrm{C}$. After addition of a $10 \%$ aqueous solution of sodium sulfite $(25 \mathrm{ml})$, stirring was maintained at the same temperature for $15 \mathrm{~min}$. The organic phase was separated and washed with a saturated solution of sodium bicarbonate $(3 \times 15 \mathrm{ml})$ and with brine $(30 \mathrm{ml})$. The organic phase was dried over anhydrous $\mathrm{MgSO}_{4}$ and the solvent was removed under vacuum. Column chromatography of the crude product $(2.5 \% \mathrm{v} / \mathrm{v}$ triethylamine-pretreated silica gel, hexane-ethyl acetate mixtures as eluents) gave $760 \mathrm{mg}(91 \%$ yield) of (2S,3S)-3-(benzyloxycarbonyl-(2-propynyl)amino)-3-phenyl-1-phenylsulfonyl-2propanol 11d as a colorless semi-solid. $[\alpha]_{\mathrm{D}}^{23}=+53.5\left(c=1.0, \mathrm{CHCl}_{3}\right) . \mathrm{IR}(\mathrm{NaCl}$ film $): v_{\max }=$ 3491, 3292, 3032, 1698, 1497, 1448, 1408, 1306, 1253, 1142, 1085, $748 \mathrm{~cm}^{-1}$. ${ }^{1} \mathrm{H}-\mathrm{NMR}$ (400 $\mathrm{MHz}_{\mathrm{CDCl}}$ ): $\delta=1.97$ (br s, 1H), 3.36-3.65 (m, 4H), 3.98 (br m, 1H), $5.20(\mathrm{~m}, 3 \mathrm{H}), 7.36-7.93$ (m, 15H) ppm. ${ }^{13} \mathrm{C}-\mathrm{NMR}\left(100 \mathrm{MHz}, \mathrm{CDCl}_{3}\right): \delta=34.1\left(\mathrm{CH}_{2}\right), 60.1\left(\mathrm{CH}_{2}\right), 63.4(\mathrm{CH}), 66.2(\mathrm{CH})$, $68.1\left(\mathrm{CH}_{2}\right), 72.4(\mathrm{CH}), 79.8(\mathrm{C}), 127.7(\mathrm{CH}), 127.9(\mathrm{CH}), 128.1(\mathrm{CH}), 128.2(\mathrm{CH}), 128.5(\mathrm{CH})$, $128.7(\mathrm{CH}), 129.0(\mathrm{CH}), 129.3(\mathrm{CH}), 134.4(\mathrm{CH}), 135.5(\mathrm{C}), 136.2(\mathrm{C}), 140.1(\mathrm{C}), 155.6(\mathrm{C})$ ppm. MS (CI, $\left.\mathrm{NH}_{3}\right)$ m/e: 481 ([M+18] $\left.]^{+}, 71 \%\right), 464\left([\mathrm{M}+1]^{+}, 100 \%\right), 420\left([\mathrm{M}-43]^{+}, 28 \%\right), 373$ ([M-90] $]^{+}, 46 \%$ ). HRMS (CI, $\left.\mathrm{NH}_{3}\right) \mathrm{C}_{26} \mathrm{H}_{26} \mathrm{NO}_{5} \mathrm{~S}(\mathrm{M}+1)$ : 464.1532 calcd., 464.1532 found.

(3S,E)-3-(Benzyloxycarbonyl-(2-propynyl)amino)-1-phenylsulfonyl-1-butene $\quad$ (2c). A solution of the hydroxysulfone $11 \mathrm{c}(0.48 \mathrm{~g}, 1.2 \mathrm{mmol})$, the water-soluble carbodiimide morpho- 
CDI (1.0 g, $2.4 \mathrm{mmol}$ ) and anhydrous copper(II) chloride (16 $\mathrm{mg}, 0.12 \mathrm{mmol})$ in dry acetonitrile $(50 \mathrm{ml})$ was heated to $70^{\circ} \mathrm{C}$ and stirred during $20 \mathrm{~h}$. After cooling to room temperature, the reaction mixture was filtered through a short pad or Celite ${ }^{\circledR}$ and silica gel, that was thoroughly washed with $\mathrm{CH}_{2} \mathrm{Cl}_{2}$. Removal of solvents under vacuum gave $450 \mathrm{mg}$ (98\% yield) of (3S,E)-3(benzyloxycarbonyl-(2-propynyl)amino)-1-phenylsulfonyl-1-butene 2c as a colorless semi-solid. $[\alpha]_{\mathrm{D}}{ }^{23}=-28.4\left(c=1.0, \mathrm{CHCl}_{3}\right)$. IR $(\mathrm{NaCl}$ film $): v_{\max }=3276,2935,1701,1447,1409,1318$, 1258, 1148, 1086, 753, $689 \mathrm{~cm}^{-1} .{ }^{1} \mathrm{H}-\mathrm{NMR}\left(400 \mathrm{MHz}, \mathrm{CDCl}_{3}\right): \delta=1.41(\mathrm{~d}, J=6.8 \mathrm{~Hz}, 3 \mathrm{H}), 2.16$ (s, 1H), 3.98 (br d, 2H), 5.03 (br m, 1H), 5.15 (s, 2H), 6.39 (d, J=15.0 Hz, 1H), 7.01 (dd, $J_{1}=$ $\left.15.0 \mathrm{~Hz}, J_{2}=4.4 \mathrm{~Hz}, 1 \mathrm{H}\right), 7.31-7.88(\mathrm{~m}, 10 \mathrm{H})$ ppm. ${ }^{13} \mathrm{C}-\mathrm{NMR}\left(100 \mathrm{MHz}, \mathrm{CDCl}_{3}\right): \delta=16.7$ $\left(\mathrm{CH}_{3}\right), 33.9\left(\mathrm{CH}_{2}\right), 51.9(\mathrm{CH}), 67.8\left(\mathrm{CH}_{2}\right), 72.1(\mathrm{CH}), 79.8(\mathrm{C}), 127.7(\mathrm{CH}), 127.8(\mathrm{CH}), 128.2$ $(\mathrm{CH}), 128.5(\mathrm{CH}), 129.3(\mathrm{CH}), 131.3(\mathrm{CH}), 133.5(\mathrm{CH}), 135.9(\mathrm{C}), 140.0(\mathrm{C}), 145.6(\mathrm{CH}), 155.1$ (C) ppm. $\mathrm{MS}\left(\mathrm{CI}, \mathrm{NH}_{3}\right)$ m/e: $401\left([\mathrm{M}+18]^{+}, 100 \%\right), 384\left([\mathrm{M}+1]^{+}, 18 \%\right), 340\left([\mathrm{M}-43]^{+}, 21 \%\right)$. HRMS (CI, $\left.\mathrm{NH}_{3}\right) \mathrm{C}_{21} \mathrm{H}_{22} \mathrm{NO}_{4} \mathrm{~S}(\mathrm{M}+1)$ : 384.1270 calcd., 384.1266 found.

(3S,E)-3-(Benzyloxycarbonyl-(2-propynyl)amino)-3-phenyl-1-phenylsulfonyl-1-propene

(2d). A solution of the hydroxysulfone $11 d(0.70 \mathrm{~g}, 1.5 \mathrm{mmol})$, the water-soluble carbodiimide morpho-CDI (1.30 g, $3.0 \mathrm{mmol})$ and anhydrous copper(II) chloride (21 mg, $0.12 \mathrm{mmol})$ in dry acetonitrile $(50 \mathrm{ml})$ was heated to $70^{\circ} \mathrm{C}$ and stirred during $1 \mathrm{~h}$. After cooling to room temperature, the reaction mixture was filtered through a short pad or Celite ${ }^{\circledR}$ and silica gel, that was thoroughly washed with $\mathrm{CH}_{2} \mathrm{Cl}_{2}$. Removal of solvents under vacuum afforded $680 \mathrm{mg}$ (quantitative yield) of pure (3S,E)-3-(benzyloxycarbonyl-(2-propynyl)amino)-3-phenyl-1phenylsulfonyl-1-propene $2 d$ as a colorless dense oil. $[\alpha]_{\mathrm{D}}^{23}=+43.7\left(c=1.0, \mathrm{CHCl}_{3}\right)$. IR $(\mathrm{NaCl}$ film): $v_{\max }=3290,3062,1703,1497,1448,1406,1319,1253,1148,1086,750,699 \mathrm{~cm}^{-1} .{ }^{1} \mathrm{H}-$ $\operatorname{NMR}\left(400 \mathrm{MHz}, \mathrm{CDCl}_{3}\right): \delta=2.11(\mathrm{~s}, 1 \mathrm{H}), 3.65\left(\mathrm{dd}, J_{1}=18.4 \mathrm{~Hz}, J_{2}=2.4 \mathrm{~Hz}, 1 \mathrm{H}\right), 4.03(\mathrm{br} \mathrm{m}$, $1 \mathrm{H}), 5.15$ (s, 2H), 6.06 (br m, 1H), $6.59\left(\mathrm{dd}, J_{1}=15.2 \mathrm{~Hz}, J_{2}=1.6 \mathrm{~Hz}, 1 \mathrm{H}\right), 7.24-7.91(\mathrm{~m}, 16 \mathrm{H})$ ppm. ${ }^{13} \mathrm{C}-\mathrm{NMR}\left(100 \mathrm{MHz}, \mathrm{CDCl}_{3}\right): \delta 34.1\left(\mathrm{CH}_{2}\right), 60.1(\mathrm{CH}), 68.1\left(\mathrm{CH}_{2}\right), 72.4(\mathrm{CH}), 79.8(\mathrm{C})$, $127.7(\mathrm{CH}), 127.9(\mathrm{CH}), 128.1(\mathrm{CH}), 128.2(\mathrm{CH}), 128.5(\mathrm{CH}), 128.7(\mathrm{CH}), 129.0(\mathrm{CH}), 129.3$ $(\mathrm{CH}), 133.2(\mathrm{CH}), 133.5(\mathrm{CH}), 135.7(\mathrm{C}), 140.1(\mathrm{C}), 142.4(\mathrm{CH}), 155.8(\mathrm{C}) \mathrm{ppm} . \mathrm{MS}\left(\mathrm{CI}, \mathrm{NH}_{3}\right)$ $m / e: 463\left([\mathrm{M}+18]^{+}, 100 \%\right), 446\left([\mathrm{M}+1]^{+}, 7 \%\right)$. HRMS (CI, NH$\left.{ }_{3}\right) \mathrm{C}_{26} \mathrm{H}_{24} \mathrm{NO}_{4} \mathrm{~S}(\mathrm{M}+1): 446.1426$ calcd., 446.1416 found.

\section{General procedures for the intramolecular Pauson-Khand reaction}

Method A: with $\mathrm{Co}_{2}(\mathbf{C O})_{8}$ and thermal activation. To a solution of $\mathrm{Co}_{2}(\mathrm{CO})_{8}(45 \mathrm{mg}, 0.13$ mmol, 1.3 equiv) in dry toluene $(2 \mathrm{ml})$, at room temperature and under argon atmosphere, a solution of enyne $\mathbf{2 b}$-d $(0.10 \mathrm{mmol})$ in dry toluene $(2 \mathrm{ml})$ was added dropwise. The resulting redcolored solution was stirred at room temperature for $30 \mathrm{~min}$, and heated to reflux until complete disappearance of the dicobalt-enyne complex (TLC monitoring). After cooling to room temperature, the reaction mixture was filtered through Celite ${ }^{\circledR}$, and washed with toluene. The solvent was removed under vacuum and the residue was purified by column chromatography (silica gel, hexanes-ethyl acetate mixtures as eluent). 


\begin{tabular}{|c|c|}
\hline Enyne & Products (\% yield) \\
\hline $2 \mathbf{b}$ & $11 \mathrm{mg} \mathbf{1 2 b}(27), 6 \mathrm{mg} \mathbf{1 3} \mathbf{b}$ (14) \\
\hline $2 \mathrm{c}$ & $5 \mathrm{mg} \mathrm{12c} \mathrm{(6),} 5 \mathrm{mg} \mathrm{13c} \mathrm{(6)}$ \\
\hline $2 d$ & $22 \mathrm{mg} \mathbf{1 2 d}(27), 16 \mathrm{mg} \mathbf{1 4} \mathbf{d}(19)$ \\
\hline
\end{tabular}

Method B: with $\mathrm{Co}_{2}(\mathrm{CO})_{8}$ and activation with amine $\boldsymbol{N}$-oxides. To a solution of $\mathrm{Co}_{2}(\mathrm{CO})_{8}(43$ $\mathrm{mg}, 0.12 \mathrm{mmol})$ in anhydrous toluene $(2 \mathrm{ml})$, at room temperature and under argon atmosphere, a solution of enyne $\mathbf{2 b - d}(0.10 \mathrm{mmol})$ in dry toluene $(2 \mathrm{ml})$ was added dropwise. The resulting redcolored solution was stirred at room temperature for $30 \mathrm{~min}$, and the amine $\mathrm{N}$-oxide $(0.78$ mmol) was added in one portion. Stirring was maintained until complete disappearance of the dicobalt-enyne complex (TLC monitoring), and the reaction mixture was filtered through Celite $^{\mathbb{R}}$, and washed with toluene. The solvent was removed under vacuum and the residue was purified by column chromatography (silica gel, hexanes-ethyl acetate mixtures as eluent).

\begin{tabular}{|c|c|c|}
\hline Enyne & $N$-oxide & Products ( $\%$ yield) \\
\hline $2 \mathrm{~b}$ & $\mathrm{NMO}$ & 9 mg 12b (22) \\
\hline $2 \mathbf{b}$ & TMANO & 17 mg 12b (24) \\
\hline 2d & TMANO & $8 \mathrm{mg} \mathbf{1 2 d}(8), 8 \mathrm{mg} \mathbf{1 4 d}(8)$ \\
\hline
\end{tabular}

Method C: with $\operatorname{Mo}(\mathbf{C O})_{6}$. To a solution of $\mathrm{Mo}(\mathrm{CO})_{6}(32 \mathrm{mg}, 0.12 \mathrm{mmol})$ and of enyne $\mathbf{2 b}-\mathbf{d}$ $(0.10 \mathrm{mmol})$ in anhydrous toluene $(5 \mathrm{ml})$, at room temperature and under argon atmosphere, DMSO (36 $\mu \lambda, 0.50 \mathrm{mmol})$ was added in one portion. The resulting solution was heated to reflux until complete disappearance of the starting enyne (TLC monitoring). After cooling to room temperature, the reaction mixture was filtered through $\mathrm{Celite}^{\circledR}$, and washed with toluene. The solvent was removed under vacuum and the residue was purified by column chromatography (silica gel, hexanes-ethyl acetate mixtures as eluents).

\begin{tabular}{|c|c|}
\hline Enyne & Products (\% yield) \\
\hline 2b & $10 \mathrm{mg} \mathrm{12b}(14), 40 \mathrm{mg} \mathrm{13b}(56)$ \\
\hline 2c & $22 \mathrm{mg} \mathrm{12c}(26), 44 \mathrm{mg} \mathrm{13c}(51)$ \\
\hline 2d & $62 \mathrm{mg} \mathrm{12d}(65)$ \\
\hline
\end{tabular}

$(1 R, 2 S, 8 R)-N$-(Benzyloxycarbonyl)-2-(tert-butyldiphenylsilyloxy)propyl-8-phenylsulfonyl-3azabicyclo[3.3.0] oct-5-en-7-one 12b. $[\alpha]_{\mathrm{D}}{ }^{23}=+38.4\left(c=1.0, \mathrm{CHCl}_{3}\right)$. IR $(\mathrm{NaCl}$ film $): v_{\max }=$ 2931, 1702, 1654, 1447, 1409, 1310, 1152, 1111, 1084, $702 \mathrm{~cm}^{-1} .{ }^{1} \mathrm{H}-\mathrm{NMR}\left(400 \mathrm{MHz}, \mathrm{CDCl}_{3}\right): \delta$ $=1.07(\mathrm{~s}, 9 \mathrm{H}), 1.60(\mathrm{~m}, 2 \mathrm{H}), 1.74(\mathrm{~m}, 2 \mathrm{H}), 3.71(\mathrm{br}, 4 \mathrm{H}), 3.85(\mathrm{~d}, J=3.2 \mathrm{~Hz}, 1 \mathrm{H}), 4.17(\mathrm{~d}, J=$ $16.4 \mathrm{~Hz}, 1 \mathrm{H}), 4.52(\mathrm{br} \mathrm{d}, 1 \mathrm{H}), 5.11(\mathrm{~s}, 2 \mathrm{H}), 5.99(\mathrm{~s}, 1 \mathrm{H}), 7.31-7.40(\mathrm{~m}, 12 \mathrm{H}), 7.54(\mathrm{t}, J=8.0 \mathrm{~Hz}$, $2 \mathrm{H}), 7.66-7.70(\mathrm{~m}, 4 \mathrm{H}), 7.93(\mathrm{~d}, J=7.2 \mathrm{~Hz}, 2 \mathrm{H}) \mathrm{ppm} .{ }^{13} \mathrm{C}-\mathrm{NMR}\left(100 \mathrm{MHz}, \mathrm{CDCl}_{3}\right): \delta=19.2$ (C), $26.2\left(\mathrm{CH}_{2}\right), 26.9\left(\mathrm{CH}_{3}\right), 29.7\left(\mathrm{CH}_{2}\right), 48.1\left(\mathrm{CH}_{2}\right), 48.7(\mathrm{CH}), 62.2(\mathrm{CH}), 64.0\left(\mathrm{CH}_{2}\right), 67.5$ $\left(\mathrm{CH}_{2}\right), 72.5(\mathrm{CH}), 124.7(\mathrm{CH}), 127.6(\mathrm{CH}), 127.7(\mathrm{CH}), 128.2(\mathrm{CH}), 128.3(\mathrm{CH}), 128.6(\mathrm{CH})$, 
$129.2(\mathrm{CH}), 129.5(\mathrm{CH}), 134.2(\mathrm{C}), 134.4(\mathrm{CH}), 135.6(\mathrm{CH}), 136.0(\mathrm{C}), 137.8(\mathrm{C}), 154.8(\mathrm{C})$, $176.5(\mathrm{C}), 195.3(\mathrm{C}) \mathrm{ppm}$. MS (CI, $\left.\mathrm{NH}_{3}\right)$ m/e: $711\left([\mathrm{M}+18]^{+}, 35 \%\right)$.

(1S,2S,8S)- $\mathrm{N}$-(Benzyloxycarbonyl)-2-(tert-butyldiphenylsilyloxy)propyl-8-phenylsulfonyl-3azabicyclo[3.3.0]oct-5-en-7-one (13b). ${ }^{1} \mathrm{H}-\mathrm{NMR}\left(400 \mathrm{MHz}, \mathrm{CDCl}_{3}\right): \delta=0.98(\mathrm{~s}, 9 \mathrm{H}), 1.42-1.64$ (br m, 4H), 3.50-3.66 (m, 2H), $3.84(\mathrm{~d}, J=4.0 \mathrm{~Hz}, 1 \mathrm{H}), 3.92(\mathrm{~m}, 1 \mathrm{H}), 4.15(\mathrm{~m}, 1 \mathrm{H}), 4.39$ (br, $1 \mathrm{H}), 4.52(\mathrm{~m}, 1 \mathrm{H}), 5.14(\mathrm{~s}, 2 \mathrm{H}), 6.03 / 6.05(\mathrm{~s}, 1 \mathrm{H}), 7.32-7.40(\mathrm{~m}, 11 \mathrm{H}), 7.53-7.57(\mathrm{~m}, 6 \mathrm{H}), 7.67$ $(\mathrm{t}, J=7.2 \mathrm{~Hz}, 1 \mathrm{H}), 7.94(\mathrm{~d}, J=7.2 \mathrm{~Hz}, 2 \mathrm{H}) \mathrm{ppm} .{ }^{1} \mathrm{H}-\mathrm{NMR}\left(300 \mathrm{MHz}, d_{6}\right.$-DMSO, 60 $\left.{ }^{\circ} \mathrm{C}\right): \delta=$ 0.99 (s, 9H), 1.29 (br, 2H), 1.42 (br, 2H), 3.56 (br s, 2H), 3.94 (br m, 2H), 4.18 (br, 1H), 4.45 (d, $J=4.2 \mathrm{~Hz}, 1 \mathrm{H}), 4.52$ (br d, 1H), $5.10(\mathrm{~s}, 2 \mathrm{H}), 6.19(\mathrm{~s}, 1 \mathrm{H}), 7.34-7.46(\mathrm{~m}, 11 \mathrm{H}), 7.57-67$ (m, 7H), $7.95(\mathrm{~d}, J=7.2 \mathrm{~Hz}, 2 \mathrm{H}) \mathrm{ppm} .{ }^{13} \mathrm{C}-\mathrm{NMR}\left(100 \mathrm{MHz}, \mathrm{CDCl}_{3}\right): \delta=19.2(\mathrm{C}), 26.2\left(\mathrm{CH}_{2}\right), 26.9$ $\left(\mathrm{CH}_{3}\right), 29.7\left(\mathrm{CH}_{2}\right), 46.1\left(\mathrm{CH}_{2}\right), 49.2 / 49.9(\mathrm{CH}), 67.0(\mathrm{CH}), 63.3\left(\mathrm{CH}_{2}\right), 67.7 / 68.0\left(\mathrm{CH}_{2}\right)$, 69.8/69.9 (CH, CHS), $124.8(\mathrm{CH}), 127.8(\mathrm{CH}), 128.2(\mathrm{CH}), 128.3(\mathrm{CH}), 128.5(\mathrm{CH}), 129.2$ $(\mathrm{CH}), 129.4(\mathrm{CH}), 129.8(\mathrm{CH}), 134.2(\mathrm{C}), 134.4(\mathrm{CH}), 135.6(\mathrm{CH}), 136.0 / 136.2(\mathrm{C}), 138.1 / 138.2$ (C), 154.8/155.3 (C), 179.1/179.5 (C), 195.3 (C) ppm. MS (CI, NH $)_{3}$ m/e: $694\left([\mathrm{M}+1]^{+}, 21 \%\right)$.

(1R,2S,8R)-N-(Benzyloxycarbonyl)-2-methyl-8-phenylsulfonyl-3-azabicyclo[3.3.0]oct-5-en-

7-one (12c). $[\alpha]_{\mathrm{D}}{ }^{23}=+78.4\left(c=0.25, \mathrm{CHCl}_{3}\right)$. IR ( $\mathrm{NaCl}$ film): $v_{\max }=2927,1700,1447,1409$, 1353, 1310, 1150, 1084, $1027 \mathrm{~cm}^{-1} .{ }^{1} \mathrm{H}-\mathrm{NMR}\left(400 \mathrm{MHz}, \mathrm{CDCl}_{3}\right): \delta=1.65(\mathrm{~d}, J=5.6 \mathrm{~Hz}, 3 \mathrm{H})$, 3.56 (br, 1H), $3.63(\mathrm{~m}, 1 \mathrm{H}), 3.87(\mathrm{~d}, J=3.6 \mathrm{~Hz}, 1 \mathrm{H}), 4.32$ (d, $J=16.4 \mathrm{~Hz}, 1 \mathrm{H}), 4.32$ (br d, 1H), $5.15(\mathrm{~m}, 2 \mathrm{H}), 5.99(\mathrm{~s}, 1 \mathrm{H}), 7.33-7.40(\mathrm{~m}, 5 \mathrm{H}), 7.60(\mathrm{t}, J=7.6 \mathrm{~Hz}, 2 \mathrm{H}), 7.71(\mathrm{t}, J=7.6 \mathrm{~Hz}, 1 \mathrm{H})$, $7.96(\mathrm{~d}, J=7.6 \mathrm{~Hz}, 2 \mathrm{H}) \mathrm{ppm} .{ }^{13} \mathrm{C}-\mathrm{NMR}\left(100 \mathrm{MHz}, \mathrm{CDCl}_{3}\right): \delta=25.2\left(\mathrm{CH}_{3}\right), 47.7\left(\mathrm{CH}_{2}\right), 52.8$ $(\mathrm{CH}), 58.8(\mathrm{CH}), 67.4\left(\mathrm{CH}_{2}\right), 72.3(\mathrm{CH}), 124.5(\mathrm{CH}), 128.1(\mathrm{CH}), 128.3(\mathrm{CH}), 128.6(\mathrm{CH})$, $129.2(\mathrm{CH}), 129.4(\mathrm{CH}), 134.5(\mathrm{CH}), 136.2(\mathrm{C}), 137.6(\mathrm{C}), 155.2(\mathrm{C}), 176.3(\mathrm{C}), 195.8(\mathrm{C}) \mathrm{ppm}$. MS (CI, $\left.\mathrm{CH}_{4}\right) m / e: 412\left([\mathrm{M}+1]^{+}, 68 \%\right), 368\left([\mathrm{M}-43]^{+}, 46 \%\right)$. HRMS (ESI-TOF) $\mathrm{C}_{22} \mathrm{H}_{22} \mathrm{NO}_{5} \mathrm{~S}$ $(\mathrm{M}+1): 412.1219$ calcd., 412.1230 found.

\section{(1S,2S,8S)-N-(Benzyloxycarbonyl)-2-methyl-8-phenylsulfonyl-3-azabicyclo[3.3.0]oct-5-en-}

7-one (13c). $[\alpha]_{\mathrm{D}}{ }^{23}=-66.0\left(c=1.0, \mathrm{CHCl}_{3}\right)$. IR $(\mathrm{NaCl}$ film $): v_{\max }=2927,1702,1654,1447$, 1414, 1355, 1310, 1268, 1154, 1084, $756 \mathrm{~cm}^{-1} .{ }^{1} \mathrm{H}-\mathrm{NMR}\left(400 \mathrm{MHz}, \mathrm{CDCl}_{3}\right): \delta=0.97 / 0.99$ (d, $J=6.0 \mathrm{~Hz}, 3 \mathrm{H}), 3.84 / 3.87(\mathrm{~d}, J=4.8 \mathrm{~Hz}, 1 \mathrm{H}), 3.89 / 3.96(\mathrm{br}, 1 \mathrm{H}), 4.22 / 4.26(\mathrm{~d}, J=4.4 \mathrm{~Hz}, 1 \mathrm{H})$, 4.37-4.49 (m, 2H), $5.17(\mathrm{~m}, 2 \mathrm{H}), 6.08 / 6.09(\mathrm{~s}, 1 \mathrm{H}), 7.36-7.39(\mathrm{~m}, 5 \mathrm{H}), 7.59(\mathrm{t}, J=7.6 \mathrm{~Hz}, 2 \mathrm{H})$, $7.70(\mathrm{t}, J=7.6 \mathrm{~Hz}, 1 \mathrm{H}), 7.96(\mathrm{~d}, J=7.6 \mathrm{~Hz}, 2 \mathrm{H}) \mathrm{ppm} .{ }^{13} \mathrm{C}-\mathrm{NMR}\left(100 \mathrm{MHz}, \mathrm{CDCl}_{3}\right): \delta=$ 14.2/15.0 $\left(\mathrm{CH}_{3}\right)$, 45.4/45.6 $\left(\mathrm{CH}_{2}\right), 49.0 / 49.4(\mathrm{CH}), 52.9(\mathrm{CH}), 67.3 / 67.5\left(\mathrm{CH}_{2}\right), 69.4 / 69.6(\mathrm{CH})$, 125.2/125.3 (CH), 128.0/128.1 (CH), $128.3(\mathrm{CH}), 128.5 / 128.6(\mathrm{CH}), 129.0 / 129.1(\mathrm{CH}), 129.3$ $(\mathrm{CH}), 134.4(\mathrm{CH}), 136.2(\mathrm{C}), 137.6 / 137.9(\mathrm{C}), 153.9 / 154.2(\mathrm{C}), 177.5 / 177.9(\mathrm{C}), 196.2(\mathrm{C}) \mathrm{ppm}$. MS (CI, $\left.\mathrm{CH}_{4}\right) \mathrm{m} / \mathrm{e}: 412\left([\mathrm{M}+1]^{+}, 71 \%\right), 368\left([\mathrm{M}-43]^{+}, 35 \%\right)$. HRMS (ESI-TOF) $\mathrm{C}_{22} \mathrm{H}_{22} \mathrm{NNaO}_{5} \mathrm{~S}$ $(\mathrm{M}+23)$ : 434.1028 calcd., 434.1033 found.

(1R,2R,8R)-N-(Benzyloxycarbonyl)-2-phenyl-8-phenylsulfonyl-3-azabicyclo[3.3.0]oct-5-en-

7-one (12d). $[\alpha]_{\mathrm{D}}^{23}=+81.7\left(c=0.25, \mathrm{CHCl}_{3}\right)$. IR (NaCl film): $v_{\max }=2926,1702,1658,1409$, 1352, 1320, 1150, 1084, 730, $699 \mathrm{~cm}^{-1} .{ }^{1} \mathrm{H}-\mathrm{NMR}\left(400 \mathrm{MHz}, \mathrm{CDCl}_{3}\right): \delta=3.81(\mathrm{~m}, 1 \mathrm{H}), 4.04(\mathrm{~d}, J$ $=3.6 \mathrm{~Hz}, 1 \mathrm{H}), 4.42(\mathrm{~d}, J=8.8 \mathrm{~Hz}, 1 \mathrm{H}), 4.55(\mathrm{~d}, J=16.2 \mathrm{~Hz}, 1 \mathrm{H}), 4.67(\mathrm{~d}, J=16.2 \mathrm{~Hz}, 1 \mathrm{H}), 4.85$ (br, 1H), $4.98(\mathrm{br}, 1 \mathrm{H}), 6.09(\mathrm{~s}, 1 \mathrm{H}), 7.21-7.31(\mathrm{~m}, 10 \mathrm{H}), 7.39(\mathrm{t}, J=8.0 \mathrm{~Hz}, 2 \mathrm{H}), 7.56(\mathrm{t}, J=7.2$ 
$\mathrm{Hz}, 1 \mathrm{H}), 7.67(\mathrm{~d}, J=8.0 \mathrm{~Hz}, 2 \mathrm{H}) \mathrm{ppm} .{ }^{13} \mathrm{C}-\mathrm{NMR}\left(100 \mathrm{MHz}, \mathrm{CDCl}_{3}\right): \delta=48.3\left(\mathrm{CH}_{2}\right), 55.6(\mathrm{CH})$, $66.2(\mathrm{CH}), 67.5\left(\mathrm{CH}_{2}\right), 71.7(\mathrm{CH}), 125.2(\mathrm{CH}), 126.1(\mathrm{CH}), 128.0(\mathrm{CH}), 128.1(\mathrm{CH}), 128.2$ $(\mathrm{CH}), 128.8(\mathrm{CH}), 128.9(\mathrm{CH}), 129.0(\mathrm{CH}), 129.1(\mathrm{CH}), 134.3(\mathrm{CH}), 136.0(\mathrm{C}), 137.6(\mathrm{C}), 154.8$ (C), 175.7 (C), 195.3 (C) ppm. MS (CI, NH$\left.)_{3}\right) m / e: 491\left([\mathrm{M}+18]^{+}, 100 \%\right), 474\left([\mathrm{M}+1]^{+}, 36 \%\right)$, 430 ([M-43] $\left.]^{+}, 11 \%\right)$. HRMS (ESI-TOF) $\mathrm{C}_{27} \mathrm{H}_{24} \mathrm{NO}_{5} \mathrm{~S}(\mathrm{M}+1)$ : 474.1375 calcd., 474.1370 found. (1R,2R,8S)-N-(Benzyloxycarbonyl)-2-phenyl-8-phenylsulfonyl-3-azabicyclo[3.3.0]oct-5-en-

7-one (14d). IR ( $\mathrm{NaCl}$ film): $v_{\max }=2926,1716,1657,1448,1410,1354,1288,1153,1114$, 1083, $699 \mathrm{~cm}^{-1} .{ }^{1} \mathrm{H}-\mathrm{NMR}\left(400 \mathrm{MHz}, \mathrm{CDCl}_{3}\right): \delta=3.21(\mathrm{~d}, J=4.0 \mathrm{~Hz}, 1 \mathrm{H}), 4.21 / 4.29(\mathrm{~m}, 1 \mathrm{H})$, $4.62 / 4.64(\mathrm{~s}, 2 \mathrm{H}), 5.09 / 5.20(\mathrm{~d}, J=12.0 \mathrm{~Hz}, 1 \mathrm{H}), 5.05-5.21(\mathrm{~m}, 2 \mathrm{H}), 5.36 / 5.43(\mathrm{~d}, J=8.8 \mathrm{~Hz}$, $1 \mathrm{H}), 6.09 / 6.10(\mathrm{~s}, 1 \mathrm{H}), 6.88-6.96(\mathrm{~m}, 3 \mathrm{H}), 7.20-7-36(\mathrm{~m}, 7 \mathrm{H}), 7.56(\mathrm{~m}, 2 \mathrm{H}), 7.67(\mathrm{t}, J=7.6 \mathrm{~Hz}$, $1 \mathrm{H}), 7.86(\mathrm{~d}, J=7.2 \mathrm{~Hz}, 1 \mathrm{H}) \mathrm{ppm} .{ }^{13} \mathrm{C}-\mathrm{NMR}\left(100 \mathrm{MHz}, \mathrm{CDCl}_{3}\right): \delta=47.7 / 47.9\left(\mathrm{CH}_{2}\right), 50.4 / 50.9$ $(\mathrm{CH}), 61.6(\mathrm{CH}), 67.5 / 67.9\left(\mathrm{CH}_{2}\right), 70.1 / 70.4(\mathrm{CH}), 126.1 / 126.2(\mathrm{CH}), 126.4(\mathrm{CH}), 127.5(\mathrm{CH})$, $128.1(\mathrm{CH}), 128.5(\mathrm{CH}), 128.6(\mathrm{CH}), 128.8(\mathrm{CH}), 129.4(\mathrm{CH}), 129.5(\mathrm{CH}), 134.6(\mathrm{CH})$, 136.2/136.9 (C), 137.5 (C), 138.1/138.5 (C), 154.1/154.6 (C), 175.8/176.5 (C), 196.0 (C) ppm. $\mathrm{MS}\left(\mathrm{CI}, \mathrm{NH}_{3}\right)$ m/e: $491\left([\mathrm{M}+18]^{+}, 100 \%\right), 474\left([\mathrm{M}+1]^{+}, 32 \%\right), 430\left([\mathrm{M}-43]^{+}, 14 \%\right)$.

\section{Acknowledgments}

This work was supported by the Ministerio de Ciencia y Tecnología (DGI, project BQU200303426). A. P. thanks the University of Barcelona for a predoctoral fellowship.

\section{References and Footnotes}

1. For leading reviews on the Pauson-Khand reaction, see: (a) Pauson, P. L. In: Organometallics in Organic Synthesis. Aspects of a Modern Interdisciplinary Field; de Meijere, A.; Dieck, T. H., Eds.; Springer: Berlin, 1988; p. 233. (b) Schore, N. E. Org. React. 1991, 40, 1. (c) Brummond, K. M.; Kent, J. L. Tetrahedron 2000, 56, 3263. (d) BlancoUrgoiti, J.; Añorbe, L.; Pérez-Serrano, L.; Domínguez, G.; Pérez-Castells, J. Chem. Soc. Rev. 2004, 33, 32. (d) Gibson, S. E.; Mainolfi, N. Angew. Chem. Int. Ed. 2005, 44, 3022. (e) Laschat, S.; Becheanu, A.; Bell, T.; Baro, A. Synlett 2005, 2547.

2. Recent examples on the application of the intramolecular Pauson-Khand reaction to the stereocontrolled synthesis of complex polycyclic systems: (a) Wilson, M. S.; Woo, J. C. S.; Dake, G. R. J. Org. Chem. 2006, 71, 4237. (b) Werner, S.; Iyer, P. S.; Fodor, M. D.; Coleman, C. M.; Twining, L. A.; Mitasev, B.; Brummond, K. M. J. Comb. Chem. 2006, 8, 368. (c) Strübing, D.; Neumann, H.; Hübner, S.; Klaus, S.; Beller, M. Tetrahedron 2005, 61, 11345. (d) James, P.; Felpin, F.-X.; Landais, Y.; Schenk, K. J. Org. Chem. 2005, 70, 7985. (e) Ghosh, S. K.; Hsung, R. P.; Liu, J. J. Am. Chem. Soc. 2005, 127, 8260. (f) Winkler, J. D.; Lee, E. C. Y.; Nevels, L. I. Org. Lett. 2005, 7, 1489. (g) Lanver, A.; Schmalz, H.-G. Eur. J. 
Org. Chem. 2005, 1444. (h) Tang, Y.; Zhang, Y.; Dai, M.; Luo, T.; Deng, L.; Chen, J.; Yang, Z. Org. Lett. 2005, 7, 885. (i) Tanimori, S.; Sunami, T.; Fukubayashi, K.; Kirihata, M. Tetrahedron 2005, 61, 2481.

3. Boñaga, L. V. R.; Krafft, M. E. Tetrahedron 2004, 60, 9795.

4. (a) Rivero, M. R.; Adrio, J.; Carretero, J. C. Eur. J. Org. Chem. 2002, 2881. (b) Adrio, J.; Rivero, M. R.; Carretero, J. C. Chem. Eur. J. 2001, 7, 2435.

5. (a) Exon, C. ; Magnus, P. J. Am. Chem. Soc. 1983, 105, 2477. (b) Magnus, P.; Exon, C.; Albaugh-Robertson, P. Tetrahedron 1985, 24, 5861.

6. (a) Castejón, P.; Pastó, M.; Moyano, A.; Pericàs, M. A.; Riera, A. Tetrahedron Lett. 1995, 36, 3019. (b) Castejón, P.; Moyano, A.; Pericàs, M. A.; Riera, A. Tetrahedron 1996, 52, 7063. (c) Castejón, P.; Moyano, A.; Pericàs, M. A.; Riera, A. Chem. Eur. J. 1996, 2, 1001. (d) Aguilar, N.; Moyano, A.; Pericàs, M. A.; Riera, A. J. Org. Chem. 1998, 63, 3560. (e) Aguilar, N.; Moyano, A.; Pericàs, M. A.; Riera, A. Tetrahedron Lett. 1999, 40, 3913. (f) Aguilar, N.; Moyano, A.; Pericàs, M. A.; Riera, A. Tetrahedron Lett. 1999, 40, 3917. (g) Catasús, M.; Moyano, A.; Pericàs, M. A.; Riera, A. Tetrahedron Lett. 1999, 40, 9309.

7. Picó, A.; Moyano, A.; Pericàs, M. A. J. Org. Chem. 2003, 68, 5075.

8. Peptidyl vinyl sulfones have been shown to be potent and selective inhibitors both of cysteine proteases and of the proteasome. See: (a) Palmer, J. T.; Rasnick, D.; Klaus, J. L.; Brömme, D. J. Med. Chem. 1995, 38, 3913. (b) Bogyo, M.; McMaster, J. S.; Gaczynska, M.; Tortorella, D.; Goldberg, A. L.; Ploegh, H. Proc. Natl. Acad. Sci. USA 1997, 94, 6629. (c) Olson, J. E.; Lee, G. K.; Semenov, A.; Rosenthal, P. J. Bioorg. Med. Chem. 1999, 7, 633. (d) Lecaille, F.; Kaleta, J.; Brömme, D. Chem. Rev. 2002, 102, 4459.

9. Jeong, W.; Yoo, S.; Lee, J. S.; Lee, R. Y.; Chung, Y. K. Tetrahedron Lett. 1991, 32, 2137.

10. (a) Hanson, R. M.; Sharpless, K. B. J. Org. Chem. 1986, 51, 1922; (b) Gao, Y.; Hanson, R. M.; Klunder, J. M.; Ko, S. Y.; Masamune, H.; Sharpless, K. B. J. Am. Chem. Soc. 1987, 109, 5765; (c) Katsuki, T.; Martín, V. S. Org. React. 1996, 48, 1.

11. Caron, M.; Carlier, P. R.; Sharpless, K. B. J. Org. Chem. 1988, 53, 5185.

12. Buchwald, S. L.; Hicks, F. A. In: Comprehensive Asymmetric Catalysis, vol. II; Jacobsen, E. N.; Pfaltz, A. ; Yamamoto, H., Eds.; Springer: Berlin, Heidelberg, 1999; p. 491.

13. Arias, J. L.; Cabrera, A.; Sharma, P.; Rosas, N.; Sampere, R. J. Mol. Cat. A: Chemical 2006, 246, 237.

14. Berk, S. C.; Grosman, R. B.; Buchwald, S. L. J. Am. Chem. Soc. 1993, 115, 4912.

15. (a) Goettmann, F.; Le Floch, P.; Sanchez, C. Chem. Commun. 2006, 180. (b) Gokel, G. W.; Marquarding, D.; Ugi, I. K. J. Org. Chem. 1972, 37, 3052-3058.

16. Villeneuve, K.; Riddel, N.; Jordan, R. W.; Tsui, G. C.; Tam, W. Org. Lett. 2004, 6, 4543.

17. (a) Mukai, C.; Hirose, T.; Teramoto, S.; Kitagaki, S. Tetrahedron 2005, 61, 10983; (b) Brummond, K. M.; Chen, H.; Fischer, K. D.; Kerekes, A. D.; Rickards, B.; Sill, P. C.; Geib, S. Org. Lett. 2002, 4, 1931; (c) Kwong, F. Y.; Lee, H. W.; Qiu, L.; Lam, W. H.; Li, Y.-M.; Kwong, H. L.; Chan, A. S. C. Adv. Synth. Catal. 2005, 347, 1750. 
18. Shibata, T.; Toshida, N.; Yamasaki, M.; Maekawa, S.; Takagi, K. Tetrahedron 2005, 61, 9974.

19. Mukai, C.; Uchiyama, M.; Hanaoka, M. J. Chem. Soc., Chem. Commun. 1992, 1014.

20. Jeong, N.; Lee, J. S.; Lee, B. Y.; Chung, Y. K. Tetrahedron Lett. 1993, 34, 2407.

21. Brummond, K. M.; Curran, D. P.; Mitasev, B.; Fischer, S. J. Org. Chem. 2005, 70, 1745.

22. (a) Rios, R.; Pericàs, M. A.; Moyano, A.; Maestro, M. A.; Mahía, J. Org. Lett. 2002, 4, 1205; (b) Rios, R.; Paredes, S.; Pericàs, M. A.; Moyano, A. J. Organomet. Chem. 2005, 690, 358.

23. Adrio, J.; Rivero, M. R.; Carretero, J. C. Org. Lett. 2005, 7, 431.

24. Rivero, M. R.; Carretero, J. C. J. Org. Chem. 2003, 68, 2975.

25. Fonquerna, S.; Rios, R.; Moyano, A.; Pericàs, M. A.; Riera, A. Eur. J. Org. Chem. 1999, 3459.

26. (a) Shambayati, S.; Crowe, W. E.; Schreiber, S. L. Tetrahedron Lett. 1990, 31, 5289; (b) Jeong, N.; Chung, Y. K.; Lee, B. Y.; Lee, S. H.; Yoo, J.-E. Synlett 1991, 204; (c) PérezSerrano, L.; Casarrubias, L.; Domínguez, L.; Pérez-Castells, J. J. Org. Chem. 2000, 65, 3513.

27. In a single experiment, the cyclization of $2 \mathbf{c}$ in the presence of the $\operatorname{Mo}(\mathrm{CO})_{3}(\mathrm{DMF})_{3}$ complex $^{23}$ afforded a 25:75 mixture of $12 \mathrm{c}$ and 13c with a $47 \%$ global yield.

28. Montaña, A. M.; Moyano, A.; Pericàs, M. A.; Serratosa, F. Tetrahedron 1985, 41, 5995.

29. (a) Castro, J.; Moyano, A.; Pericàs, M. A.; Riera, A. Tetrahedron 1995, 51, 6541; (b) Verdaguer, X.; Vázquez, J.; Fuster, G.; Bernardes-Génisson, V.; Greene, A. E.; Moyano, A.; Pericàs, M. A.; Riera, A. J. Org. Chem. 1998, 63, 7037; (c) Vázquez, J.; Fonquerna, S.; Moyano, A.; Pericàs, M. A.; Riera, A. Tetrahedron: Asymmetry 2001, 12, 1837. (d) Pericàs, M. A.; Balsells, J.; Castro, J.; Marchueta, I.; Moyano, A.; Riera, A.; Vázquez, J.; Verdaguer, X. Pure Appl. Chem. 2002, 74, 167.

30. Pastó, M.; Moyano, A.; Pericàs, M. A.; Riera, A. Tetrahedron: Asymmetry 1995, 6, 2329.

31. Dale, J. A.; Dull, D. L.; Mosher, H. S. J. Org. Chem. 1969, 34, 2543.

32. Wang, Y. F.; Dumas, D. P.; Wong, C. H. Tetrahedron Lett. 1993, 34, 403.

33. Matsumura, Y.; Shiozawa, T.; Matsushita, H.; Yoshiyasu, T. Biol. Pharm. Bull. 1995, 18, 1805.

34. Hoffman, R.V.; Weiner, W.S.; Maslouh, N. J. Org. Chem. 2001, 66, 5790. 